\title{
Partitioning of Tetrachlorophenol into Lipid Bilayers and Sarcoplasmic Reticulum: Effect of Length of Acyl Chains, Carbonyl Group of Lipids and Biomembrane Structure
}

\author{
R.C. Word, P. Smejtek \\ Department of Physics, Portland State University, Portland, OR 97207, USA
}

Received: 23 September 2004/Revised: 4 February 2005

Abstract. We report results of a partitioning study of 2,3,4,6-tetrachlorophenol (TeCP). In the study we explored (1) the effect of the length of acyl chains of lipids (C16:1 - C24:1) and alkanes (C6-C16), (2) the role of the carbonyl group of lipids, and (3) the effect of molecular structure of the sarcoplasmic reticulum membrane on TeCP partitioning. Mole fraction partition coefficients have been measured using equilibrium dialysis for un-ionized (HA), and ionized (A) species, $K p_{\mathrm{x}}^{\mathrm{HA}}, K p_{\mathrm{x}}^{\mathrm{A}}$. Their values are concentrationdependent. Partition coefficients were analyzed in terms of a model that accounts for saturation of membrane associated with the finite area of partition site, and electrostatic interactions of (A-) species with charged membrane. Limiting values of partition coefficients, corresponding to infinite dilution of solute, $K p_{\mathrm{x} 0}^{\mathrm{HA}}, K p_{\mathrm{x} 0}^{\mathrm{A}}$ were obtained. $K p_{\mathrm{x} 0}^{\mathrm{HA}}$ and $K p_{\mathrm{x} 0}^{\mathrm{A}}$ measure the strength of solute-membrane interactions. Studies were done with single-layered vesicles of lipids with variable chain length: 1,2-dipalmitoleoyl-sn-glycero-3phosphocholine (C16:1), 1,2-dioleoyl-sn-glycero-3phosphocholine (C18:1), 1,2-dierucoyl-sn-glycero-3phosphocholine (C22:1), and 1,2-dinervonoyl-sn-glycero-3-phosphocholine (C24:1), and egg-PC. $K p_{\mathrm{x} 0}$ for transfer of TeCP from water into lipid membranes was found to be independent of the length of acyl chains, whereas $K p_{\mathrm{x} 0}$ for transfer from water into alkanes increased with the length of alkane. The effect of the carbonyl $\mathrm{CO}$ group of lipids on partitioning was measured using 1,2-di-o-octadecenyl-sn-glycero-3phosphocholine (CO absent) and 1,2-dioleoyl-sn-glycero-3-phosphocholine (CO present) liposomes. Carbonyl groups, known to change dipolar potential, had no effect on partitioning. Partition coefficients of unionized and ionized forms of $\mathrm{TeCP}$ were invariant to the presence of proteins and other membrane components of sarcoplasmic reticulum (SR) membrane.
Key words: Tetrachlorophenol - Partition coefficient - Dipolar potential - Acyl chains - Carbonyl group - Ionic strength effect - Liposomes Sarcoplasmic reticulum

\section{Introduction}

In the present, "post-Overton" period (Kieinzeller, 1999; Cantor, 2001), it is becoming increasingly recognized that (1) partitioning of small molecules between water and disordered and ordered immiscible media is more complex than previously thought, especially at the molecular level (Smejtek \& Word, 2004), and (2) that more detailed experimental results are needed to understand partition and predict behavior of small molecules in living systems (White \& Wimley, 1999). A very useful and unifying hypothesis of membrane activity of amphiphilic molecules, such as TeCP, was formulated by Herbette and Gruner in (Herbette \& Gruner 1987). It is concerned with mode of action of drugs mediated by lipid matrix. It is applicable to cases in which a lipid matrix acts as a reservoir and concentrator of solutes due to their high partition coefficients. The membrane-bound lipophilic molecules laterally diffuse within the lipid matrix of biomembrane acting as a reservoir and bring about an increased probability of specific or non-specific interactions with receptor proteins. In addition, their presence alters physicalchemical properties of membranes and results in altered biological response. Membrane concentrations of biologically active molecules have been often estimated from their octanol-water partition coefficients. However, in some instances octanol-water partition coefficients cannot be used as predictors of biological response, as shown by Herbette, Chester and Rhodes, because they are not applicable to the water-membrane system (Herbette, et al., 1986). These issues have been reviewed by Seydel (Seydel, 2002). 
Biological activity of TeCP is due to induced transmembrane transfer of protons (Escher, Smozzi \& Schwatzenbach, 1996; Escher, Hunziket and Schwarzenback, 1999). In this mode of action the lipid bilayer also acts as a concentrator and facilitates passive proton transport at low aqueous concentrations of TeCP. We selected TeCP for its suitable physicochemical properties. The focus of the present work is accurate measurement of partition coefficients and of the partition properties of TeCP in water-lipid bilayer and water-sarcoplasmic reticulum systems. In these studies we used an equilibrium dialysis method complemented by spectrophotometric determination of initial and equilibrium concentrations. An excellent discussion of experimental methods of measuring partition coefficients, with emphasis on optical spectroscopy, was published by Santos, Prieto and Castanho (Santos, et al. 2003). Another excellent review of the use of liposomes in studies of partitioning of small molecules and drugs, focused on drug research, was published recently by Kramer (Kramer, 2001) and by Testa and coworkers (Plemper van Balen, et al., 2004).

In the present work we studied (1) the effect of length of acyl chains on partitioning of TeCP between water and alkanes, and between water and lipid membranes, (2) the effect of carbonyl group of lipids and (3) the effect of structure of biological membrane and proteins on partitioning of TeCP into sarcoplasmic reticulum membrane. In this work, sarcoplasmic reticulum is regarded as a reference biological membrane. Furthermore, since under biologically relevant $\mathrm{pH}$ 's $\mathrm{TeCP}$ is present both as unionized (HA) and ionized (A-) species, and both species are biologically active, we studied membrane partition properties of both.

We describe partitioning in terms of the mole fraction partition coefficients for several reasons. One is that the number of lipids and the number of membrane-bound TeCP molecules are directly measured. Another reason is that mole fraction partition coefficients are dimensionless quantities. The thermodynamic significance of mole fraction partition coefficients has been debated in the literature (Tanford, 1979; Ben-Naim, 1979). However, in membrane studies, use of mole fraction partition coefficients allow us to avoid the problem of uncertainty in membrane volume in which membrane-bound TeCP molecules are preferentially located. Membranes cannot be regarded as thin layers of homogeneous medium.

Due to the great hydrophobicity of TeCP one cannot exclude the possibility that TeCP molecules are preferentially located within the region of acyl chains of lipids. Since unionized TeCP (HA) molecules may concentrate in the environment of alkane chains, one might expect that they dissolve in the membrane core region, i.e., within acyl chains of lipids. This is the least dense portion of the membrane, with the largest fraction of free volume and therefore the lowest work required to create a cavity in which $\mathrm{TeCP}$ can be localized. For reference we measured partition coefficients for transfer of TeCP from water into alkanes. Since the alkane-water partition coefficient increased with the number of alkane carbons, we were interested in finding whether or not the mole fraction partition coefficient $K p_{\mathrm{x}}$ of un-ionized TeCP would increase with the length of acyl chains of lipids in the following order $K p_{\mathrm{x}}(\mathrm{DPOPC})<K p_{\mathrm{x}}(\mathrm{DOPC})<K p_{\mathrm{x}}(\mathrm{DEPC})<$ $K p_{\mathrm{x}}(\mathrm{DNPC})$. We found that the partition coefficient of TeCP in lipid bilayers was in fact independent of the length of lipid acyl chains. This is in contrast to partition into alkanes where the mole fraction partition coefficient of un-ionized TeCP increases with alkane length. We conclude that partition sites of un-ionized $\mathrm{TeCP}$ are localized in lipid membranes. In contrast, TeCP partitions into alkanes unrestrictedly, as if alkane phase were a free volume.

The ionized species (A-) are known to be less hydrophobic. Because of their negative charge, we expect TeCP ions to be localized within the membrane, close to the membrane/water interface, like other hydrophobic ions (Smejtek \& Wang, 1990; Smejtek, 1995). In such a case the partition coefficient of ionized TeCP would be independent of the length of acyl chains of lipids. This was confirmed by experimental results.

We also report on the effect of the carbonyl group of lipids on partition of TeCP. It was shown that carbonyl groups in the ester linkages of acyl chains make the dipolar potential inside the membrane more positive (Gawrisch, et al., 1992) by about $120 \mathrm{mV}$. The dipolar electric field is $10-100$ times greater than the transmembrane field in biomembranes under physiological conditions. It was also shown that the dipolar potential played a dominant role in partition of positively and negatively charged lipophilic ions (Flewelling \& Hubbell, 1986). To evaluate the role of the carbonyl group of lipids in partition, we have measured partition coefficients of un-ionized and ionized TeCP using DOPC and DODPC membranes that differ by the presence or absence of a carbonyl group. We found no difference.

We have also studied partitioning of TeCP between water and sarcoplasmic reticulum membrane to assess the effect of membrane proteins and molecular structure of the biomembrane. Partition properties of sarcoplasmic reticulum are compared to those of egg-PC bilayer. Thus, if SR proteins or other SR membrane components would enhance or diminish the partition of TeCP, the changes of partition relative to egg-PC would be observed.

Results of this study are relevant to toxicology, pharmacokinetics and drug design by providing the experimental values of $K p_{\mathrm{x} 0}$ for the HA and its conjugate A- species. Values of $K p_{\mathrm{x} 0}$ are also needed for 
Table 1. Materials

Materials

Abbreviation

Source

Lipids

L- $\alpha$-Phosphatidylcholine

1,2-Dipalmitoleoyl-sn-glycero-3-phosphocholine

1,2-Dioleoyl-sn-glycero-3-phosphocholine

egg-PC

DPOPC

DOPC

DEPC

DNPC

1,2-Dinervonoyl-sn-glycero-3-phosphocholine

DODPC

Avanti ${ }^{1}$

Avanti ${ }^{1}$

Avanti ${ }^{1}$

Avanti ${ }^{1}$

Avanti ${ }^{1}$

1,2-Di-o-octadecenyl-sn-glycero-3-phosphocholine

Avanti ${ }^{1}$

Buffers

Sodium acetate

acetic acid

EM Science ${ }^{2}$

2-[n-Morpholino]ethanesulfonic acid

MES

$\mathrm{n}$-[2-Hydroxyethyl]piperazine-n'-[2-ethane-sulfonic acid]

HEPES

2-[n-Cyclohexylamino]ethanesulfonic acid

CHES

Boric acid

$\mathrm{H}_{3} \mathrm{~B}_{3}$

Potassium phosphate dibasic

$\mathrm{K}_{2} \mathrm{HPO}_{4}$

Potassium citrate

$\mathrm{K}_{3} \mathrm{C}_{6} \mathrm{H}_{5} \mathrm{O}_{7}$

Aldrich $^{3}$

Aldrich $^{3}$

Aldrich $^{3}$

Mallinckrodt ${ }^{4}$

$\mathrm{MCB}^{5}$

$\mathrm{MCB}^{5}$

Alkanes

Hexane

Heptane

Octane

Decane

Dodecane

Tetradecane

Hexadecane

Aldrich $^{3}$

Aldrich $^{3}$

Aldrich $^{3}$

Aldrich $^{3}$

Aldrich $^{3}$

Aldrich $^{3}$

Aldrich $^{3}$

Other compounds

2,3,4,6-Tetrachlorophenol

$\mathrm{TeCP}$

$\mathrm{TCI}^{6}$

SM-2 Adsorbent

Bio-Beads

$\mathrm{BioRad}^{7}$

$\mathrm{MCB}^{8}$

Ammonium molybdate tetrahydrate

Octylphenoxy polyethoxyethanol

Triton X-100

Aldrich $^{3}$

${ }^{1}$ Avanti Polar Lipids, Inc., Alabaster Alabama; ${ }^{2}$ EM Science, Gibbstown, New Jersey; ${ }^{3}$ Aldrich, Milwaukee, WI; ${ }^{4}$ Mallinckrodt, St. Louis, Missouri; ${ }^{5}$ Matheson Coleman \& Bell Manufacturing Chemists, Norwood, Ohio; ${ }^{6}$ TCl America, Portland, Oregon; ${ }^{7}$ Bio Rad Laboratories, Hercules, California ${ }^{8}$ Matheson Coleman \& Bell Manufacturing Chemists, Norwood, Ohio. All compounds were used as obtained from the manufacturer.

adjusting parameters in molecular models. This study contributes to the biophysical understanding of interaction of small molecules with lipid bilayers and biological membranes. An important implication is that, at least for TeCP and possibly other small hydrophobes, the lipid bilayer is a very good model for biological membranes.

\section{Materials and Methods}

Lipids, buffers, alkanes, other compounds, and their abbreviations are listed in Table 1.

\section{Liposomes and Sarcoplasmic Reticulum Vesicles}

Single-layered lipid vesicles (SLV) were produced using a detergent extraction method. Lipids dissolved in chloroform in a roundbottom flask were dried using a rotary evaporator and dried under vacuum overnight. The lipids were then dissolved in buffer $(100 \mathrm{~mm}$ $\mathrm{KCl}$ and $10 \mathrm{~mm}$ acetic acid $\left(p K_{\mathrm{a}}=4.76\right), \operatorname{MES}\left(p K_{\mathrm{a}}=6.21\right)$, HEPES $\left(p K_{\mathrm{a}}=7.66\right)$, or CHES $\left.\left(p K_{\mathrm{a}}=9.44\right)\right)$ and Triton X-100 ( $80 \mu \mathrm{L}$ Trixton X-100 per $1 \mathrm{~mL}$ buffer per $40 \mathrm{mg}$ of lipids). Triton $\mathrm{X}-100$ was removed in three stages using three columns of Bio Beads SM2 $(1 \mathrm{~mL}$ of Bio Beads per $1 \mathrm{~mL}$ of solution for each stage). The sequence of stages lasted $1 \mathrm{~h}, 1 \mathrm{~h}$, and $30 \mathrm{~min}$. The suspension was removed from each column by mild centrifugation. These conditions are similar to those used for preparation of proteoliposomes (Paucek, et al., 1992). The original procedure for extracting nonionic Triton X-100 by BioBeads was developed by Holloway (Holloway, 1973). The mechanism and optimization of conditions for preparation of liposomes and proteoliposomes using polystyrene, such as Bio Beads SM2 are discussed and reviewed by Rigaud (Rigaud, et al., 1998).

Sarcoplasmic reticulum vesicles were kindly provided locally by professor Jon Abramson. They were prepared from rabbit skeletal muscle as outlined in Xia, et al., (2003).

\section{Determination of Lipid Content of Liposome/SR SUSPENSIONS}

The lipid content of suspensions was determined by measuring their phosphate content using the "ascorbic acid method" described by Chen (Chen, Toribara \& Warner, 1956). In this procedure, liposome or SR suspensions are dried, ashed with sulfuric acid, and oxidized under flame with $30 \%$ hydrogen peroxide to obtain inorganic phosphate. When mixed with sulfuric acid and ammonium molybdate, phosphate forms a complex, $\left(\mathrm{NH}_{4}\right)_{3}\left[\mathrm{PO}_{4}\left(\mathrm{MoO}_{3}\right)_{12}\right]$ which is yellow to blue, depending on its concentration. The extinction coefficient of this complex was found to be $27,100 \pm 100 \mathrm{M}^{-1} \mathrm{~cm}^{-1}$ at $820 \mathrm{~nm}$. Concentration of lipids in this study is assumed to be equal to the concentration of phosphomolybdate complex. 


\section{Paritioning into Liposome and Sarcoplasmic Reticulum}

The partition coefficient was determined using the equilibrium dialysis method in combination with spectrophotometric determination of concentration of TeCP using a Beckman DU-7 spectrophotometer (Irvine, CA). Our method is an extension of the method used by Escher (Escher, et al. 1996). In the present study, three distribution experiments were performed concurrently. Each experiment required a measurement cell and a lipid-free reference cell. Two additional control cells were common to the three pairs of measurement and reference cells. The first of the control cells contained liposomes or SR vesicles and buffer. This cell is designated as the lipid or SR reference cell. The second cell contained only buffer and is designated as the background cell. One experimental run used a total of eight dialysis cells: one background, one lipid or SR reference, three reference, and three measurement cells. The dialysis cells were each made of two blocks of machined Teflon. Since un-ionized TeCP sorbed to Teflon, we used the reference and background cells to make the accurate determination of partition coefficients possible. The compartments were separated by cellulose membrane with a molecular weight cutoff of $6,000-8,000$ (Cellu-Sep T2 from Membrane Filtration Products, Inc., Texas). The volume of liquid in each compartment was $5.00 \mathrm{ml}$. Except in the ionic-strength dependence experiments, the composition of aqueous phase (also referred to as buffer) was $0.100 \mathrm{M} \mathrm{KCl}$ and $10.0 \mathrm{mM}$ of acetic acid, MES, HEPES, or CHES.

The dialysis cells were prepared by injecting liposome suspension (or SR), TeCP solution, or buffer as shown in Table 2. All of the dialysis cells were shaken overnight (about $20 \mathrm{~h}$ ) on a Burrell Wrist-Action Shaker (Pittsburgh, PA). By varying the equilibration time we established that $20 \mathrm{~h}$ of equilibration was more than adequate. After shaking, samples from each chamber were extracted using a long narrow disposable pipette and kept in labeled sample bottles. The equilibrium concentration of TeCP was measured spectrophotometrically after converting all TeCP present in the sample into its ionized form (extinction coefficient $=5,010 \pm 20 \mathrm{M}^{-1} \mathrm{~cm}^{-1}$ at $316.0 \mathrm{~nm}$ ).

This conversion maximizes the accuracy of the spectrophotometric method. The absorbance spectra of samples from (measurement, reference, and lipid reference cells were measured in the range from 250.0 to $450.0 \mathrm{~nm}$ three times each against the background cell. The absorbance of the lipid reference was subtracted from the measurement cell. Additionally, 1.00 to $4.00 \mathrm{ml}$ of each liposome sample was saved in 30 -mL test tubes for later measurement of their lipid concentration.

\section{Partitioning into Alkanes}

We used the traditional shake-flask method to obtain alkane-water partition coefficients. We filled $50-\mathrm{mL}$ glass centrifuge tubes with $25.00 \mathrm{~mL}$ of buffer and $5.00 \mathrm{~mL}$ of a 10 to $20 \mathrm{mM} \mathrm{TeCP}$ alkane solution (at least three flasks for each alkane). The buffer was 2.0 $\mathrm{mM} \mathrm{K}_{2} \mathrm{PO}_{4}, 2.0 \mathrm{mM} \mathrm{H}_{3} \mathrm{BO}_{3}, 5.0 \mathrm{mM} \mathrm{K}_{3} \mathrm{C}_{6} \mathrm{H}_{5} \mathrm{O}_{7}$, and $0.030 \mathrm{M}$ $\mathrm{KCl}$ buffer adjusted to $\mathrm{pH} 3.0$ with $\mathrm{HCl}$. The flasks were shaken by hand and placed in a $20.0^{\circ} \mathrm{C}$ water bath for $20 \mathrm{~h}$. Afterwards, the alkane phase was removed by pipette and discarded. Samples from the water phases were adjusted to $\mathrm{pH}>10$ with $\mathrm{KOH}$ and their spectra measured in the spectrophotometer. The partition coefficients were determined using a mass balancing approach. It follows that the equilibrium molar concentration of $\mathrm{TeCP}$ in the alkane phase, $C_{\text {eq,alk }}$, is equal to

$C_{\text {eq,alk }}=C_{\text {o,alk }} \frac{V_{\text {water }}}{V_{\text {alk }}} C_{\text {eq,water }}$.
Table 2. Contents of the dialysis cells

\begin{tabular}{llll}
\hline Cell & Liposomes/SR & TeCP solution & Buffer solution \\
\hline Measurement & $5.00 \mathrm{~mL}$ & $5.00 \mathrm{~mL}$ & - \\
Reference & - & $5.00 \mathrm{~mL}$ & $5.00 \mathrm{~mL}$ \\
Lipid reference & $5.00 \mathrm{~mL}$ & - & $5.00 \mathrm{~mL}$ \\
Background & - & - & $2 \times 5.00 \mathrm{ml}$ \\
\hline
\end{tabular}

Here $C_{\text {o,alk }}$ is the initial molar concentration of TeCP in alkane, $V_{\text {water }}$ is the volume of the water phase, $V_{\text {alk }}$ is the volume of the alkane phase, and $C_{\text {eq,water }}$ is the equilibrium molar concentration of TeCP in the water phase determined from its spectrum. The mole fraction partition coefficient, $K p_{\mathrm{x}}$, defined as the equilibrium mole fraction of $\mathrm{TeCP}$ in alkane to that in water, is then

$$
\begin{aligned}
& K p_{\mathrm{x}}=\frac{x_{\mathrm{alk}}}{x_{\mathrm{water}}}=\frac{\frac{C_{\mathrm{eq}, \mathrm{alk}}}{C_{\mathrm{eq}, \mathrm{alk}}+C_{\mathrm{alkane}}}}{\frac{C_{\mathrm{eq} \text { water }}}{C_{\mathrm{eq}, \mathrm{water}}+C_{\mathrm{water}}}} \cong \frac{C_{\mathrm{eq}, \mathrm{alk}}}{C_{\mathrm{eq}, \mathrm{water}}} \cdot \frac{C_{\mathrm{water}}}{C_{\mathrm{alkane}}} \\
& \quad C_{\text {alkane and } C_{\mathrm{water}} \text { are the molar concentrations of each }} \\
& \text { solvent in their own phases. }
\end{aligned}
$$

\section{Method of Determination of Membrane-Water PARTITION COEFFICIENTS}

Experimental values of mole fraction membrane-water partition coefficients were determined according to

$K p_{\mathrm{x}}=\frac{x_{\mathrm{m}}}{x_{\mathrm{aq}}}$

where $x_{\mathrm{m}}$ is the equilibrium mole fraction of membranebound TeCP and $x_{\mathrm{aq}}$ is the equilibrium mole fraction of TeCP in water. These are

$$
x_{\mathrm{m}}=\frac{C_{\text {bound }}}{C_{\text {bound }}+C_{\text {lipid }}}
$$

and

$$
x_{\mathrm{aq}}=\frac{C_{\text {eq,water }}}{C_{\text {water }}+C_{\text {eq,water }}}
$$

Experimental partition coefficients were calculated according to

$$
K p_{\mathrm{x}}(\exp )=\frac{\mathrm{C}_{\text {bound }}}{\frac{V_{1}}{V_{1}+V_{2}} \mathrm{C}_{\text {lipid }}+C_{\text {bound }}} \frac{1}{x_{\mathrm{aq}}} .
$$

$V_{1}$ is the volume of the lipid-containing compartment and $V_{2}$ is the volume of the other compartment of the dialysis cell. The concentration of lipids is equal to concentration of phosphomolybdate complex in the liposome suspension. $C_{\text {bound }}$ is the effective molar concentration of TeCP bound to the vesicles in the suspension. It is proportional to the difference between the reference and equilibrium TeCP concentrations.

$C_{\text {bound }}=\left(1+\frac{\Gamma_{\mathrm{H}}}{1+10^{\mathrm{pH}-\mathrm{pKa}}}\right)\left(C_{r e f}-C_{e q, \text { water }}\right)$.

represents the loss of un-ionized TeCP due to sorption to Teflon and cellulose membrane. Sorption of ionized TeCP was not significant. The pKa of 2,3,4,6-TeCP is 5.4 (Schellenberg, et al., 1984). In all membrane-partitioning studies the quantity $C_{\text {eq, water }}$ will be further simplified by $C_{\text {eq }}$. 


\section{Correction Method Used to Incorporate Dialysis}

\section{Cell Sorption into Partition Coefficient}

\section{DeterminATION}

The amount of TeCP bound to liposomes ( $\left.\mathrm{N}_{\text {bound }}\right)$ must be determined from measurement of the aqueous concentration of TeCP in measurement cell and the lipid-free reference cell. Loss of TeCP to the dialysis cell walls and the cellulose membrane occurs in each of the cells. Below we present a method of accounting for this loss in the calculation of $\mathrm{N}_{\text {bound }}$.

\section{Reference Cell}

The concentration of TeCP in the reference cell, $C_{\text {ref }}$, may be less than the total concentration, $C_{\mathrm{o}}$, originally injected into the cell due to the loss in the cell, $N_{\text {loss. }}$. If $V$ is the volume of the dialysis cell, then the mass-balance for the reference cell yields

$$
C_{\mathrm{o}} V=C_{\text {ref }} V+N_{\text {loss }} \text {. }
$$

In a dialysis experiment involving liposomes we cannot assume that loss in the measurement cell is equal to the loss in the reference cell $/ N_{\text {loss. }}$. We need a method to estimate $N_{\text {loss }}$ in the measurement cell from the concentration in the reference cell. We assume that the mechanism of loss of TeCP to the dialysis cell walls and cellulose membrane surfaces follows Henry's law, i.e., $N_{\text {loss }}$ is linearly proportional to the equilibrium concentration, $C_{\mathrm{ref}}$,

$N_{\text {loss }}=\frac{\Gamma_{\mathrm{H}}}{1+10^{p H-p K a}} \cdot C_{r e f} \cdot V$.

The loss coefficient $\Gamma_{\mathrm{H}}$ was experimentally determined. From Eq. (7) follows that the total and reference concentrations are then related by

$C_{o}=\left(1+\frac{\Gamma_{\mathrm{H}}}{1+10^{p H-p K a}}\right) C_{r e f}$.

The fractional loss $\Gamma_{\mathrm{H}}$, can be determined from the measured concentrations in the reference cell,

$\Gamma_{\mathrm{H}}\left(1+10^{p H-p K a}\right)=\left(\frac{C_{\mathrm{o}}}{C_{r e f}}-1\right)$

For our cells the loss parameter $\Gamma_{\mathrm{H}}=0.143 \pm 0.022$.

\section{Measurement Cell}

To find the concentration of TeCP bound to liposomes, we start with another mass balance equation.

$C_{\mathrm{o}} V=C_{\text {eq }} V+N_{\text {bound }}+N_{\text {loss }}$.

$C_{\mathrm{o}}$ is known and the equilibrium concentration $C_{\mathrm{eq}}$ is experimentally measured. $N_{\text {bound }}$ is the number of TeCP molecules bound to liposomes. It is important to realize that TeCP bound to liposomes is not directly available for sorption to the dialysis cell. The number of TeCP molecules in water, available for sorption to the cell, $N_{\text {avail }}$, follows from be the mass balance

$C_{\mathrm{o}} V=N_{\text {avail }}+N_{\text {bound }}$.

Since the measurement cell and the reference cell are identical, the loss coefficients, $\Gamma_{\mathrm{H}}$, are the same as well,

$N_{\text {loss }}=\frac{\Gamma_{\mathrm{H}}}{1+10^{p H-p K a}} \cdot C_{e q} V$.

From the mass balance equation for the measurement cell, Eq. (11), follows that

$C_{\mathrm{o}} V=C_{e q} V+N_{\text {bound }}+\frac{\Gamma_{\mathrm{H}}}{1+10^{p H-p K a}} C_{e q} V$.

Since $C_{\text {bound }}=N_{\text {bound }} / V$, this approach makes it possible to determine $C_{\text {bound }}$ from the two experimentally determined values, the equilibrium concentration in the measurement cell, $C_{\mathrm{eq}}$ and the equilibrium concentration in the reference cell, $C_{\text {ref }}$,

$C_{\text {bound }}=\left(1+\frac{\Gamma_{\mathrm{H}}}{1+10^{p H-p K a}}\right)\left(C_{r e f}-C_{e q}\right)$.

Concentration $\mathrm{C}_{\text {bound }}$ obtained from Eq.(15) can be used to calculate the experimental value of the mole fraction partition coefficient of un-ionized species using Eq. (5). If TeCP is partially ionized, the concentration $\mathrm{C}_{\text {bound }}$ should be obtained according Eq. (6).

\section{Membrane Partition Model}

The experimental values of partition coefficient $K p_{\mathrm{x}}$ of both unionized and ionized species of TeCP were found not to be constant, but dependent upon the equilibrium concentration $C_{\text {eq }}$. In case of ionized TeCP, $K p_{\mathrm{x}}$ also depends on the ionic strength of the aqueous phase due to changes of membrane surface potential associated with screening of charged membrane surface. Since $K p_{\mathrm{x}}$ is not a constant, it is further regarded as an "apparent partition coefficient." Our objective is to obtain partition coefficients that reflect the solutemembrane interaction. This can be achieved by extrapolating the partition coefficients to limit of infinite dilution of solute

$K p_{\mathrm{x} 0}=\lim _{C_{\mathrm{eq}} \rightarrow 0} K p_{\mathrm{x}}$.

$K p_{\mathrm{x}}$ is the apparent partition coefficient obtained at experimentally accessible concentrations $C_{\text {eq }}$, whereas $K p_{\mathrm{x} 0}$ is the thermodynamically relevant quantity. The objective of this work is to determine $K p_{\mathrm{x} 0}$ for the un-ionized and the ionized TeCP for membranes of different structure.

Since it is not 'a priori' known where in the membrane TeCP is localized, we use the mole fraction partition coefficient and recognize that partition into membranes is an interfacial process in which the properties of membrane surface, such as electric charge and presence of proteins play a major role in regulating the concentration of ionized solutes. We have adopted the two-dimensional double-layer partition model illustrated in Fig.1 since the area of membrane surface per lipid molecule or per membranebound protein is known.

Partitioning of TeCP into the lipid bilayer is referenced to the surface of membrane. Local minima of Gibbs free energy of solute in the membrane are associated with partition sites. One partition site is associated with membrane surface areas $A_{\mathrm{s}}$. The membrane surface is a two-dimensional array of partition sites even though the regions of lowest free energy are not at the surface but may be in the interior of membrane. These sites can be occupied either by un-ionized (HA) or ionized (A-) TeCP species. We realize that there are no lipid-specific sites for sorption of TeCP and that partition sites are a consequence of collective interactions within the lipid array.

Membrane surface area is determined by the number of lipids, $L$, and by the area per lipid, $A_{\mathrm{L}}$. In view of the facts that both the (HA) and (A-) species are membrane-permeable, and in view of the the long time allowed for equilibration and the small curvature of liposome membrane, we assume that partition properties of both the inner and the outer surface of liposomes are the same. The number of partition sites in a given experiment is determined by the surface area of lipid membrane, $A_{\mathrm{m}}$, and the partition site area $A_{\mathrm{s}}$. For simplicity we assume that partition sites for (HA) or (A-) are the same. The number of partition sites is 


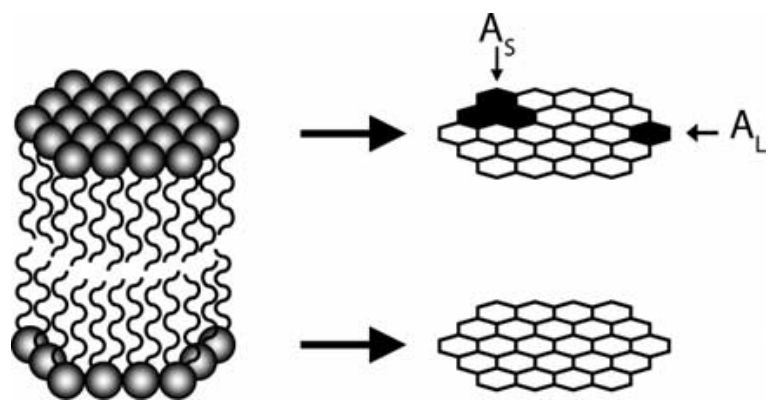

Fig. 1. Presence of TeCP in the lipid bilayer referenced to the surfaces of the membrane. Local minima of Gibbs free energy of $\mathrm{TeCP}$ in the membrane are associated with partition sites. One partition site is associated with the surface area $A_{\mathrm{s}}$. In the model the partition sites can be occupied either by un-ionized (HA) or ionized (A-) TeCP species. Membrane surface area is determined by the number of lipids, $L$, and by the area per lipid, $A_{\mathrm{L}}$.

$$
N=\frac{A_{\mathrm{m}}}{A_{\mathrm{s}}}=\frac{L A_{\mathrm{L}}}{A_{\mathrm{s}}} .
$$

$A_{\mathrm{L}}$ is the membrane surface area per lipid molecule and $L$ the number of lipids in a given experiment. The number of lipid molecules per partition site is

$\frac{L}{N}=\frac{A_{\mathrm{s}}}{A_{\mathrm{L}}}$.

The surface density of partition sites is an important quantity. It determines the saturation of the membrane and the solute concentration profile of the partition isotherm. Furthermore, if the nearest neighbor solutes are prevented from approaching each other within a distance comparable to average interlipid distance, due to lateral interaction of solutes in the membrane, the number of lipids per partition site partition sites will be greater than unity.

The fraction of partition sites occupied by solute, or the fractional coverage is equal to

$\theta=\frac{S}{N}=\frac{S}{L} \frac{A_{\mathrm{S}}}{A_{L}}$.

where $S$ is the number of solutes in the membrane and $N$ the number of partition sites (Eq. 17). The distribution of solute across the membrane/water interface results from diffusive equilibrium. It follows from the Langmuir model that the fractional coverage is

$\theta=\frac{K_{\mathrm{m}} x_{\mathrm{aq}}}{1+K_{\mathrm{m}} x_{\mathrm{aq}}}$.

$K_{\mathrm{m}}$, the equilibrium association constant of solute, is the ratio of association and dissociation rate constants of solutes entering and departing from the membrane.

When modeling the partition, we calculate the "theoretical" apparent partition coefficient

$K p_{x}(t h)=\frac{S}{S+N A_{\mathrm{S}} / A_{\mathrm{L}}} \frac{C_{\mathrm{water}}+C_{\mathrm{eq}}}{C_{\mathrm{eq}}} \cong \frac{\theta}{\theta+A_{S} / A_{\mathrm{L}}} \frac{C_{\text {water }}}{C_{e q}}$

and compare its value with $K p_{\mathrm{x}}(\exp )$ determined from the experiment according to Eq. (5).

The association constant in Langmuir isotherm $K_{\mathrm{m}}$, and the limiting value of the mole fraction partition coefficient $K p_{\mathrm{x} 0}$ are related to each other according to

$$
K_{\mathrm{m}}=K p_{x 0} \frac{A_{\mathrm{S}}}{A_{L}}
$$

This relationship follows from the definition of $K p_{\mathrm{x}}$ in combination with Eqs. (19) and (20) under the condition of low aqueous concentration of solute so that the fractional coverage is small, $\theta<<$ $A_{\mathrm{S}} / A_{\mathrm{L}}$, and the partition isotherm is linear, $K_{\mathrm{m}} X_{\mathrm{aq}}<<1$.

Using the infinite dilution mole fraction partition coefficient, $K p_{\mathrm{x} 0}$, the Langmuir partition isotherm becomes

$$
\theta=\frac{K \mathrm{p}_{x 0} C_{e q} / C_{\text {water }}}{A_{L} / A_{S}+K p_{x 0} C_{e q} / C_{\text {water }}}
$$

It incorporates both the solute-membrane interaction (Eq 22) expressed by the infinite dilution partition coefficient $K p_{\mathrm{x} 0}$ and the partial saturation of membrane at finite $C_{\text {eq }}$, the equilibrium molar concentration of solute.

At $\mathrm{pH}$ comparable to the $\mathrm{pKa}$ of TeCP $(=5.4)$, both unionized (HA) and ionized species (A-) are prominent and compete for the available partition sites. These conditions can be described in terms of fractional membrane coverage for each species. It follows from the two-component Langmuir model that the fractional coverages are, respectively,

$$
\begin{aligned}
& \theta_{\mathrm{HA}} \cong \frac{K p_{\mathrm{x} 0}^{\mathrm{HA}} C_{\text {HAEeq }} / C_{\text {water }}}{A_{\mathrm{L}} / A_{\mathrm{S}}+K p_{x 0}^{\mathrm{HA}} C_{\mathrm{HAeq}} / C_{\text {water }}+K p_{\mathrm{x} 0}^{\mathrm{A}} C_{\text {Aeq }}^{\text {if }} / C_{\text {water }}} \\
& \theta_{\mathrm{A}} \cong \frac{K p_{\mathrm{x} 0}^{\mathrm{A}} C_{\text {Aeq }}^{\text {if }} / C_{\text {water }}}{A_{\mathrm{L}} / A_{\mathrm{S}}+K p_{\mathrm{x} 0}^{\mathrm{HA}} C_{\mathrm{HAeq}} / C_{\text {water }}+K p_{\mathrm{x} 0}^{\mathrm{A}} C_{\text {Aeq }}^{\mathrm{if}} / C_{\text {water }}}
\end{aligned}
$$

Equations (24 a,b) describe the competition of (HA) and (A-) species for the membrane. The concentrations of un-ionized and ionized species can be determined from Henderson-Hasselbach relations and experimental equilibrium concentration of TeCP, $C_{\mathrm{eq}}$.

$C_{\text {HAeq }}=\frac{C_{\text {eq }}}{1+10^{\mathrm{pH}-\mathrm{pKa}}}$

$C_{\text {Aeq }}=\frac{C_{\text {eq }}}{1+10^{\mathrm{pKa}-\mathrm{pH}}}$

When ionized TeCP partitions into an electrically neutral lipid membrane, the membrane becomes charged and repels the ionized species away from the interface. This electrostatic interaction can be accounted for by a Boltzmann factor,

$C_{\text {Aeq }}^{\text {if }}=C_{\text {Aeq }} \exp \left(-z_{\mathrm{A}} e \psi / k T\right)$

The interfacial concentration of ionized TeCP, which in turn determines the partition into the membrane, depends upon the electrostatic potential $\psi$. The surface potential thus depends on the partition coefficient.

\section{Electrostatic Effects in Partitioning of Ionized Species A}

The study of the dependence of partition on the length of acyl chains was done with uncharged lipids. In such a case the membrane acquires negative charge due to the presence of ionized TeCP, A-. The surface charge density is assumed to be

$\sigma_{\mathrm{m}}=\frac{\text { charge }}{\text { area }}=\frac{S_{\mathrm{A}} z_{\mathrm{A}} e}{L A_{L}}$

where $e$ denotes unit charge. In order to relate the surface charge density to fractional coverage $\theta_{\mathrm{A}}$, we use Eq. (19) and obtain 
$\sigma_{\mathrm{m}}=\frac{S_{\mathrm{A}} z_{\mathrm{A}} e}{N A_{\mathrm{S}}}=\theta_{\mathrm{A}} \frac{z_{\mathrm{A}} e}{A_{\mathrm{S}}}$

Negative charge on the membrane attracts positively charged ions in the aqueous phase so that the charge on the membrane is screened by the space charge in aqueous phase. This condition of charge compensation is expressed in Grahame's equation (Starzak 1984). We use Grahame's equation to calculate the electrostatic potential $\psi$ at the membrane/water interface. When several types of ions, " $i$ ", of molar concentration $c$ i are present, Grahame's equation becomes

$$
\begin{array}{r}
\sqrt{2 \varepsilon_{\mathrm{W}} \varepsilon_{0} R T 1000\left(\text { liter } / m^{3}\right) \sum_{\mathrm{i}} c_{\mathrm{i}}\left[\exp \left(-z_{\mathrm{i}} e \psi / k T\right)-1\right]} \\
+\theta_{\mathrm{A}}\left(z_{\mathrm{A}} e / A_{\mathrm{S}}\right)=0
\end{array}
$$

The explicit form of Grahame's equation including the partition coefficient of ionized species is

$$
\begin{aligned}
& \sqrt{2 \varepsilon_{\mathrm{w}} \varepsilon_{0} R T 1000\left(\text { liter } / m^{3} \sum_{\mathrm{i}} c_{\mathrm{i}}\left[\exp \left(-z_{\mathrm{i}} e \psi / k T\right)-1\right]\right.} \\
& +\left(z_{\mathrm{A}} e / A_{\mathrm{S}}\right) \frac{K p_{\mathrm{x} 0}^{\mathrm{A}} C_{\mathrm{Aeq}}^{\mathrm{if}} / C_{\mathrm{water}}}{A_{\mathrm{L}} / A_{\mathrm{S}}+K p_{\mathrm{x} 0}^{\mathrm{HA}} C_{\mathrm{HAeq}} / C_{\text {water }}+K p_{\mathrm{x} 0}^{\mathrm{A}} C_{\mathrm{Aeq}}^{\mathrm{if}} / C_{\text {water }}}=0
\end{aligned}
$$

Experimental values of partition coefficients at high $\mathrm{pH}$ are most suitable for the determination of the partition coefficient of deprotonated $\mathrm{TeCP}, \mathrm{Kp} \mathrm{p}_{\mathrm{x} 0}^{\mathrm{A}}$, since the contribution of the un-ionized form can be neglected. A representative value of $K p_{\mathrm{x} 0}^{\mathrm{A}}$ is obtained from the agreement between the "theoretical" and experimental values of apparent partition coefficients,

$$
K p_{\mathrm{x}}(A, t h)=\frac{\theta_{\mathrm{A}}}{\theta_{\mathrm{A}}+A_{\mathrm{S}} / A_{\mathrm{L}}} \frac{C_{\text {water }}}{C_{\text {Aeq }}} \cong K p_{x}(A, \exp )
$$

At low $\mathrm{pH}$, partitioning of $\mathrm{TeCP}$ is dominated by the unionized species HA. Similarly, for the representative value of $K p_{\mathrm{x} 0}^{\mathrm{HA}}$,

$K p_{x}(H A, t h)=\frac{\theta_{\mathrm{HA}}}{\theta_{\mathrm{HA}}+A_{\mathrm{S}} / A_{\mathrm{L}}} \frac{C_{\mathrm{water}}}{C_{\mathrm{HAeq}}} \cong K p_{\mathrm{x}}(H A, \exp )$

At arbitrary $\mathrm{pH}$, the partition sites are occupied by both (HA) and (A-) species so that the total $\mathrm{TeCP}$ partition coefficient predicted from $K p_{\mathrm{x} 0}^{\mathrm{HA}}$ and $K p_{\mathrm{x} 0}^{\mathrm{A}}$, is in agreement with the measured value,

$K p_{x}(t h)=\frac{\theta_{\mathrm{HA}}+\theta_{\mathrm{A}}}{\theta_{\mathrm{HA}}+\theta_{\mathrm{A}}+A_{\mathrm{S}} / A_{\mathrm{L}}} \frac{C_{\mathrm{water}}}{C_{e q}} \cong K p_{\mathrm{x}}(\exp )$

where $\theta_{\mathrm{H}} \mathrm{A}$ and $\theta_{\mathrm{A}}$ are determined from Eqs. (24a) and (24b).

An alternate description of membrane saturation by the sorbed solute is by the dependence of $K p_{\mathrm{x}}$ on the mole fraction of membrane-bound TeCP, $x_{\mathrm{m}}$. For the un-ionized species the partition coefficient is a linear function of $x_{\mathrm{m}}$,

$K p_{\mathrm{x}}(H A, t h)=K p_{\mathrm{x} 0}^{\mathrm{HA}}\left[1-x_{\mathrm{m}}\left(1+A_{\mathrm{S}} / A_{\mathrm{L}}\right)\right] \cong K p_{\mathrm{x}}(H A, \exp )$

$K p_{\mathrm{x} 0}^{\mathrm{HA}}$ is the intercept of the linear fit of $K p_{\mathrm{x}}$ vs $x_{\mathrm{m}}$ at low $\mathrm{pH}$. The slope, equal to $K p_{\mathrm{x} 0}^{\mathrm{HA}}\left(1+\mathrm{As} / \mathrm{A}_{\mathrm{L}}\right)$, provides value of $A \mathrm{~s} / A_{\mathrm{L}}$.

The infinite dilution partition coefficient of ionized species, $K p_{\mathrm{x} 0}^{\mathrm{A}}$, was obtained as the intercept of the linear fit of $\log K p_{\mathrm{x}}$ vs $x_{\mathrm{m}}$. It was not possible to determine the value of $A_{\mathrm{S}} / A_{\mathrm{L}}$ for ionized TeCP from our experimental data. The reason is that the electrostatic repulsion between ionized $\mathrm{TeCP}$ in the aqueous phase and the TeCP-charged membrane rather than the saturation of partition sites dominate the curvature of sorption isotherm. Both versions of the partition model were used to interpret the experimental partition data.

\section{Results and Discussion}

\section{Effect of Acyl Chain Length on Partitioning}

To assess the effect of the length of acyl chains of lipids we measured partition coefficient of TeCP as a function of aqueous concentration of TeCP and $\mathrm{pH}$ using single-layered liposomes prepared from lipids of variable chain length. These were DPOPC (16:1), DOPC (18:1), DEPC (22:1) and DNPC (24:1). EggPC liposomes were used as a reference. Partition coefficients for liposomes were compared with partition coefficients for alkanes with chain length ranging from hexane (C6) to hexadecane (C16).

Partition coefficients of TeCP $(\mathrm{pKa}=5.4)$ were measured at $\mathrm{pH} 4.0$ or $4.1,5.5,7.0$, and 9.5. Within this range TeCP is present as predominantly un-ionized at the low $\mathrm{pH}$, to predominantly ionized at the high $\mathrm{pH}$. The equilibrium concentration of TeCP varied from $4 \mu \mathrm{M}$ to $70 \mu \mathrm{M}$. Since different methods of obtaining least square fit weigh the errors differently, the experimental partition coefficients $K p_{\mathrm{x}}$, obtained according to Eq. (5), were fitted by two versions of the partition model to obtain the infinite dilution partition coefficient, $K p_{\mathrm{x} 0}$ and the ratio of partition site to lipid surface area, $A_{\mathrm{s}} / A_{\mathrm{L}}$. In version 1 we fit the dependence of $K p_{\mathrm{x}}$ on solute equilibrium concentration, i.e., $K p_{\mathrm{x}}$ vs $C_{\text {eq. }}$. In version 2 we fit the dependence of $K p_{\mathrm{x}}$ on equilibrium membrane concentration measured by mole fraction $x_{\mathrm{m}}$, i.e., $K p_{\mathrm{x}}$ vs $x_{\mathrm{m}}$. In version $1, K p_{\mathrm{x} 0}$ is obtained from LSF of the concentration dependence of $K p_{\mathrm{x}}$, in version 2, by linear extrapolation of $K p_{\mathrm{x}}$ to $x_{\mathrm{m}}=0$. The values of $K p_{\mathrm{x} 0}$ for un-ionized TeCP (HA), obtained for $\mathrm{pH}$ 4-4.1, and for anionic form of TeCP (A-), obtained for $\mathrm{pH}$ 9.5, are listed in Table $3 A$ and $3 B$. The quality of the fit can be judged from the value of $r^{2}$ and $\chi^{2}$ (The residual of a least-squares fit. In this case, $\chi^{2}=\sum_{\mathrm{i}=1}^{\mathrm{n}}\left(K p_{\mathrm{x}}(\mathrm{th})_{\mathrm{i}}-K p_{\mathrm{x}}(\exp )_{\mathrm{i}}\right)^{2} / \sigma_{\exp }^{2}, \mathrm{i}$, where $\sigma_{\exp , \mathrm{i}}^{2}$ is the estimated variance of each data point. $\chi^{2}<n-1$ is desirable (Bevington \& Robinson, 2003) although not always achievable.) At intermediate $\mathrm{pH}$, when both (HA) and (A-) species contribute to membrane partition, the measured dependence of $K p_{\mathrm{x}}$ on equilibrium concentration agrees with the predicted one according to Eq. $(24 \mathrm{a}, \mathrm{b})$ and $(33)$. The ratio of $A_{\mathrm{S}} / A_{\mathrm{L}}$ for unionized TeCP (HA) was about 0.9 from the version 1 fit and $0.7-0.8$ from version 2 fit. It indicates that the origin of the saturation of partition isotherm is the membrane surface area of the partition site, which is slightly smaller than the membrane surface area per lipid $\left(\approx 0.7 \mathrm{~nm}^{2}\right)$. It should be pointed out that the above model and method of analysis is free of the assumption of formation of 1:1 complex between the solute and the lipid, which is frequently used in partitioning studies (Kramer, 2003). The ratio of $A_{\mathrm{S}} / A_{\mathrm{L}}$ 
Table 3. Effect of acyl chains. Least-squares-fit values of the infinite dilution partition coefficient, $K p_{\mathrm{x} 0}$, of the un-ionized (HA) and ionized (A-) species of TeCP

\begin{tabular}{|c|c|c|c|c|c|c|}
\hline Lipid & $\mathrm{p}^{\mathrm{H}}$ & $N$ & $\chi^{2}$ & $K \mathrm{p}_{\mathrm{x} 0}$ & $\mathrm{TeCP}$ & $r^{2}$ \\
\hline \multicolumn{7}{|l|}{$(A)$} \\
\hline DPOPC (16:1) & 4.0 & 6 & 2.0 & $(2.56 \pm 0.08) \times 10^{6}$ & HA & \\
\hline DPOPC (16:1) & 9.5 & 6 & 9.2 & $(0.139 \pm 0.004) \times 10^{6}$ & A- & \\
\hline DOPC (18:1) & 4.1 & 9 & 7.0 & $(2.35 \pm 0.05) \times 10^{6}$ & HA & \\
\hline DOPC (18:1) & 9.5 & 6 & 4.9 & $(0.149 \pm 0.005) \times 10^{6}$ & A- & \\
\hline DEPC (22:1) & 4.1 & 6 & 2.0 & $(2.96 \pm 0.13) \times 10^{6}$ & HA & \\
\hline DEPC (22:1) & 9.5 & 6 & 1.2 & $(0.167 \pm 0.006) \times 10^{6}$ & A- & \\
\hline DNPC (24:1) & 4.0 & 6 & 6.8 & $(2.54 \pm 0.09) \times 10^{6}$ & HA & \\
\hline DNPC (24:1) & 9.5 & 5 & 2.7 & $(0.101 \pm 0.004) \times 10^{6}$ & A- & \\
\hline Egg-PC (reference) & 4.0 & 6 & 1.4 & $(1.81 \pm 0.06) \times 10^{6}$ & HA & \\
\hline Egg-PC (reference) & 9.1 & 6 & 1.4 & $(0.1 .21 \pm 0.004) \times 10^{6}$ & A- & \\
\hline \multicolumn{7}{|l|}{$(B)$} \\
\hline DPOPC (16:1) & 4.0 & 6 & 9.3 & $(2.32 \pm 0.10) \times 10^{6}$ & HA & 0.99 \\
\hline DPOPC (16:1) & 9.5 & 6 & 6.1 & $(0.131 \pm 0.008) \times 10^{6}$ & A- & 0.93 \\
\hline DOPC (18:1) & 4.1 & 9 & 63 & $(1.86 \pm 0.08) \times 10^{6}$ & HA & 0.94 \\
\hline DOPC (18:1) & 9.5 & 6 & 0.58 & $(0.131 \pm 0.009) \times 10^{6}$ & A- & 0.99 \\
\hline DEPC (22:1) & 4.1 & 6 & 13 & $(2.45 \pm 0.16) \times 10^{6}$ & HA & 0.97 \\
\hline DEPC (22:1) & 9.5 & 6 & 1.4 & $(0.165 \pm 0.011) \times 10^{6}$ & A- & 0.99 \\
\hline DNPC (24:1) & 4.0 & 6 & 38 & $(1.98 \pm 0.11) \times 10^{6}$ & HA & 0.93 \\
\hline DNPC (24:1) & 9.5 & 5 & 2.1 & $(0.0937 \pm 0.0089) \times 10^{6}$ & A- & 0.97 \\
\hline Egg-PC (reference) & 4.0 & 6 & 1.7 & $(1.68 \pm 0.10) \times 10^{6}$ & HA & 0.93 \\
\hline Egg-PC (reference) & 9.4 & 6 & 0.32 & $(0.115 \pm 0.007) \times 10^{6}$ & A- & 0.99 \\
\hline
\end{tabular}

(A) Results obtained from the dependence of $K p_{\mathrm{x}}$ on the aqueous equilibrium concentration of TeCP. The least-squares-fit method includes Eqs. (5), (24a), (24b), (25a), (25b), (26), (30) and (33).

$(B)$ Results obtained from the dependence of $K p_{\mathrm{x}}$ on the mole fraction of bound TeCP.

determines the curvature of the dependence of $K p_{\mathrm{x}}$ on the equilibrium concentration of solute in water and its value is obtained from the least-squares-fit of the model to the $K p_{\mathrm{x}}$ data. It does not provide information on membrane swelling, only on the distance of closest approach at saturation. Although the $K p_{\mathrm{x}}$ measurements were done at $0.100 \mathrm{M} \mathrm{KCl}$, an estimate of $A_{\mathrm{s}} / A_{\mathrm{L}}$ for ionized TeCP (A-) could not be obtained because the saturation of the partition isotherm is almost entirely of electrostatic origin: the repulsion of negatively charged TeCP from the membrane surface. At higher salt concentration the electrostatic effects could be reduced but the predictions based on the GouyChapman model become less accurate, so that the ratio $A_{\mathrm{s}} / A_{\mathrm{L}}$ would not be reliable. Our overall conclusion from the statistical analysis of the dependence of $K p_{\mathrm{x}}$ on the length of acyl chains using a 2-sided $t$-test is that $K p_{\mathrm{x}}$ of un-ionized TeCP does not linearly correlate with the number of carbons in acyl chains. A plot of the dependence of $K p_{\mathrm{x}}$ on the equilibrium concentration of TeCP at $\mathrm{pH} 4.0-4.1$ (Fig. 2) shows that the present partition model and one value of the "infinite dilution" partition coefficient $K p_{\mathrm{x} 0}^{\mathrm{HA}}$ explains the partition isotherms of un-ionized TeCP for DPOPC, DOPC, DEPC and DNPC membranes.

Data in Tables $3 A, B$ suggest that the value of $K p_{\mathrm{x}}$ for ionized TeCP (A-) slightly increased with the number of chain carbons for $\mathrm{C} 16, \mathrm{C} 18$ and $\mathrm{C} 22$ chains but decreased significantly for DNPC (C24). This drop in $K p_{\mathrm{x}}$ may be caused by a small amount of negatively charged impurities in DNPC. In Fig. 2 we also illustrate the dependence of the measured $K p_{\mathrm{x}}$ on equilibrium concentration of ionized $\mathrm{TeCP}$ obtained at $\mathrm{pH}$ 9.5. The data for DPOPC, DOPC and DEPC membranes are also consistent with a single value of $K p_{\mathrm{x} 0}^{\mathrm{A}}$.

In Figure 3 we compare the dependence of partition coefficients of un-ionized TeCP on the number of carbons in acyl chains of alkanes and lipids. Overlap of partition data for alkanes and lipids is limited by the fact that longer alkanes are solids under current experimental conditions $\left(20.0{ }^{\circ} \mathrm{C}\right)$ and lipid membranes with shorter chains are unstable. Within the experimentally accessible carbon chain length range, the results for alkanes indicate that the partition coefficient of TeCP increases with the length of alkane whereas such an effect is absent for liposomes.

The magnitude of $K p_{\mathrm{x}}$ and its independence of the length of lipids illustrate both the effect of the alignment of acyl chains and the environment of the polar head groups of lipids in a liposome membrane. In alkanes, acyl chains are in disordered state. In this environment the number of methylene groups available for van-der-Waals interaction with TeCP per alkane increases with the length of acyl chain, resulting in the increase of $K p_{\mathrm{x}}$. In contrast, local environment of membrane-bound $\mathrm{TeCP}$ is entirely different. Due to the presence of polar head groups, partial penetration of water into the membrane/water 


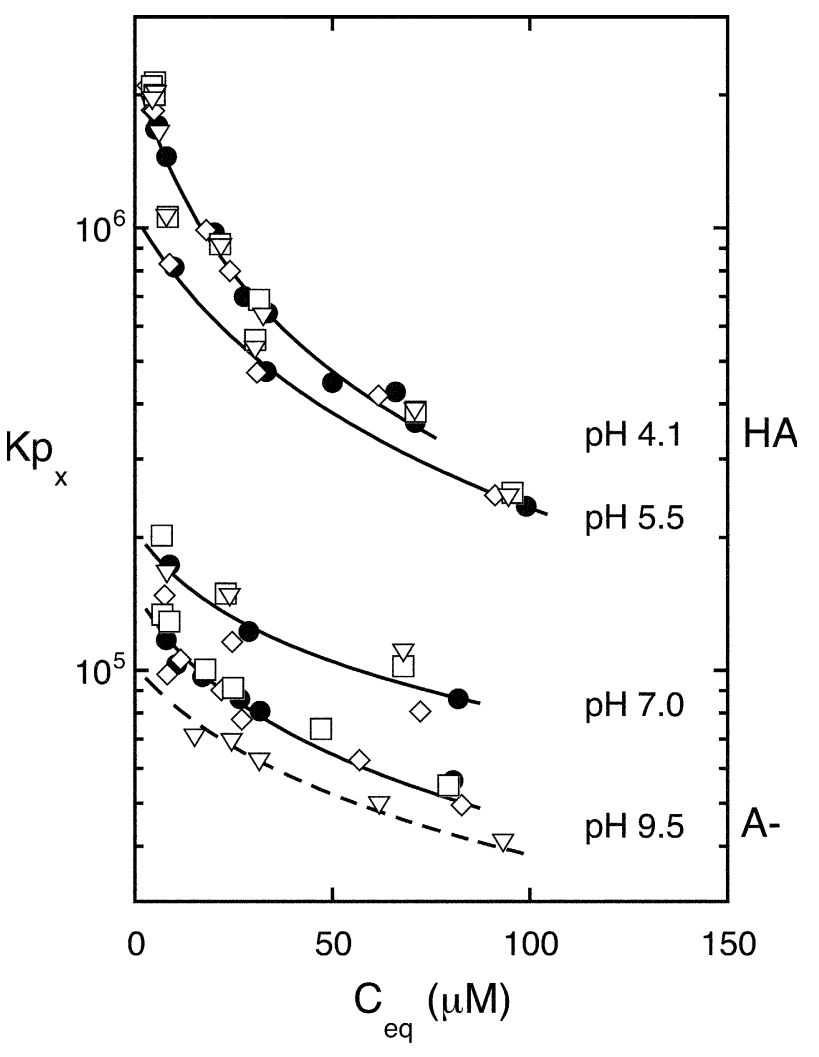

Fig. 2. Experimental results illustrating the independence of the partition coefficient of TeCP on the length of acyl chains of membrane lipids. Plots of the partition coefficient as a function of equilibrium concentration of $\mathrm{TeCP}$ for lipid bilayers with different lengths of acyl chains: DPOPC (C16 $\diamond)$, DOPC (C18 ), DEPC $(\mathrm{C} 22 \square)$, and DNPC (C24 $\nabla)$. The datasets are, from top to bottom, of experiments at $\mathrm{pH} 4.0-4.1, \mathrm{pH} 5.5, \mathrm{pH} 7.0$, and $\mathrm{pH} 9.5$. The concentration dependence of the partition coefficient for a given $\mathrm{pH}$ and lipids with different chain length can be explained with one value of $K p_{\mathrm{x} 0}$. The solid curves are theoretical, $K p_{\mathrm{x}}(t h)$, based on least-squares fits of all $K p_{\mathrm{x}}$ (exper) data at each $\mathrm{pH}$ with the exclusion of DNPC at $\mathrm{pH}$ 9.5. The partition coefficient curves, including all lipids, were calculated using $K p_{\mathrm{x} 0}^{\mathrm{HA}}=2.6 \times 10^{6}$, and $K p_{\mathrm{x} 0}^{\mathrm{A}}=0.15 \times 10^{6}$. Partition site area $A_{\mathrm{s}}=0.65 \mathrm{~nm}^{2}$ was used in all cases. The dashed curve is for DNPC calculated with $K p_{\mathrm{x} 0}^{\mathrm{A}}=0.103 \times 10^{6}$. Calculation of concentration dependence of $K p_{\mathrm{x}}(t h)$ included Eqs. (24a), (24b), (25a), (25b), (26), (30) and the left side of Eq. (33).

interface and the alignment of acyl chains, the energy of attractive interactions between $\mathrm{TeCP}$ and its environment is significantly greater in the lipid bilayer than in alkanes. Data in Fig. 3 illustrate, by extrapolation, that the change of environment from alkane into bilayer is as bilayer is associated with the increase of TeCP partition coefficient by $2-3$ orders of magnitude.

\section{EfFect of Carbonyl Groups of Lipids on TeCP PARTITIONING}

The dipolar field within the water-membrane interface has a significant effect on the rate of trans-

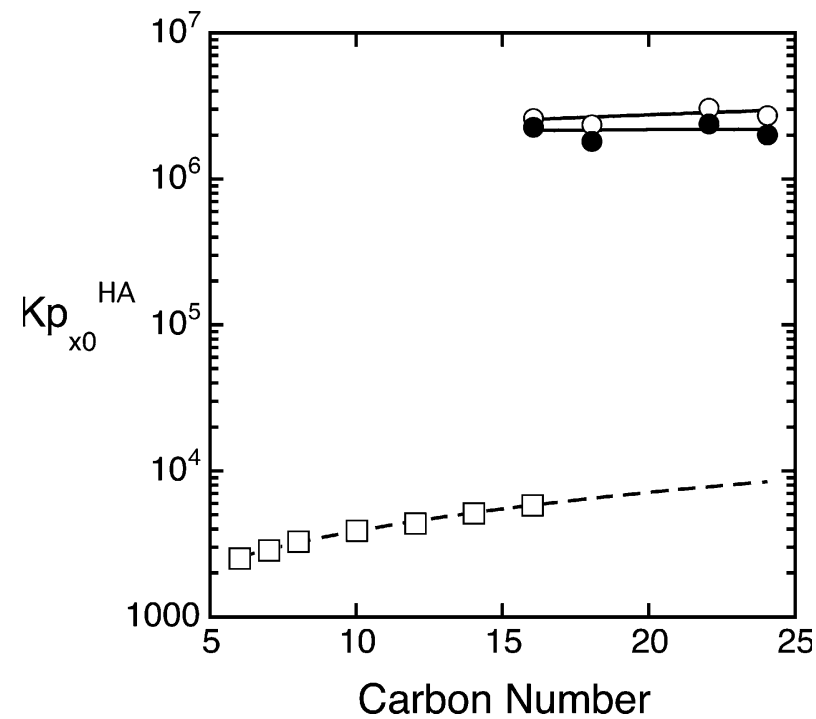

Fig. 3. Comparison of partitioning into acyl chains of alkanes and lipid bilayers with acyl chains of variable length. Dependence of partition coefficients of un-ionized TeCP, $K p_{\mathrm{x} 0}^{\mathrm{HA}}$, on number of carbons in acyl chains. The broken curve depicts the least-squaresfit dependence of the alkane partition coefficient on the number of carbons. The lower set (squares) represents results for alkanes and the upper set, for DPOPC (C16), DOPC (C18), DEPC (C22) and DNPC (C24) liposome membranes. The empty circles represent infinite dilution partition coefficients obtained using the first partition model $\left(\mathrm{Kp}_{\mathrm{x}}\right.$ vs. $\left.\mathrm{C}_{\mathrm{eq}}\right)$ and the filled circles are from fits of the second partition model $\left(\mathrm{K}_{\mathrm{x}}\right.$ vs. $\left.\mathrm{X}_{\mathrm{m}}\right)$. The continuous lines illustrate the respective linear least-squares-fits.

membrane transport of lipophilic cations and anions, as well as transport of alkali metal cations and protons through channels (Rokitskaya, et al., 2002). The effect can be understood in terms of modulation of the ion Gibbs free energy profile along the membrane conduction pathway by the interfacial dipoles. Flewelling and Hubbell have shown how dipolar potential changes the partition coefficient of lipophilic ions (Flewelling \& Hubbell, 1986).

There is a consensus in the literature that carbonyl group of the $s n_{2}$ chain of phosphatidylcholines and water polarized at the water-lipid interface are the major contributors to positive-inside dipolar potential. The dipole moment of the carbonyl group of the $s n_{2}$ chain is approximately perpendicular to the membrane surface. In contrast, the carbonyl group of the $s n_{1}$ chain is parallel to the membrane surface and does not contribute to the dipolar potential. It was shown experimentally (Gawrisch, et al., 1992) that the dipolar potential of dipalmitoylphosphatidylcholine, which contains carbonyl groups, is about 120 $\mathrm{mV}$ more positive than the dipolar potential of dihexadecylphosphatidylcholine, in which carbonyl groups are absent.

In this work we have studied the effect of carbonyl groups of lipids on membrane partitioning of both un-ionized and ionized $\mathrm{TeCP}$ by measuring partition coefficients of (HA) and (A-) species using 

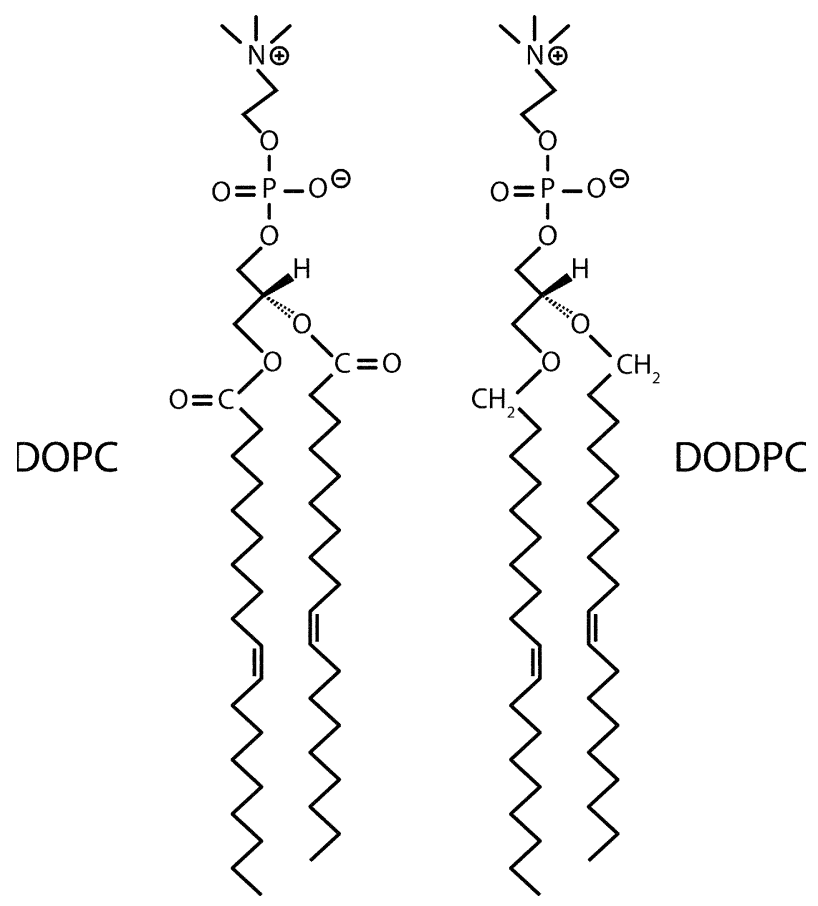

Fig. 4. Molecular structure of lipids used in the study of the effect of carbonyl groups on partitioning of TeCP, Carbonyl groups are absent in DODPC and present in DOPC.

liposomes prepared from DOPC and DODPC (Fig. 4). Carbonyl groups are present in DOPC, and absent in DODPC. If the Gibbs free energy of membrane-bound TeCP changed due to the absence of carbonyl groups in DODPC, we would observe a change of TeCP partition coefficient from that of DOPC. Change of Gibbs free energy of TeCP associated with the carbonyl group could be determined from the change of partition coefficient.

Experimental results are shown in Fig. 5. The empty symbols depict the partition coefficient obtained with DODPC liposomes, the filled symbols are for DOPC. Partition measurements were done at $\mathrm{pH}$ 4,0, 5.5, 7.0 and 9.5. At $\mathrm{pH} 4 \mathrm{TeCP}$ is $96 \%$ un-ionized (HA), whereas at $\mathrm{pH} 9.5$ it is fully $(99.99 \%)$ ionized (A-). The partition coefficient data in Fig. 5 demonstrate that there is no effect of dipolar field associated with carbonyl groups. The Gibbs free energy of the TeCP partition site in the membrane for both the (HA) and (A-) species did not change when carbonyl groups were removed. The solid curves illustrate the predictions of the partition model. Values of partition coefficients are summarized in Table 4.

The absence of the effect of carbonyl groups on partitioning of TeCP appear to contradict the known contribution of dipolar potential to the free energy of partitioning of lipophilic ions in carbonyl groupcontaining membranes (Flewelling \& Hubbell 1986). There is no doubt that the carbonyl group of $\mathrm{sn}_{2}$ chains (Gawrisch, et al. 1 992) as well as the $\mathrm{C}=\mathrm{O}$ group of 6-ketocholestanol incorporated into lipid

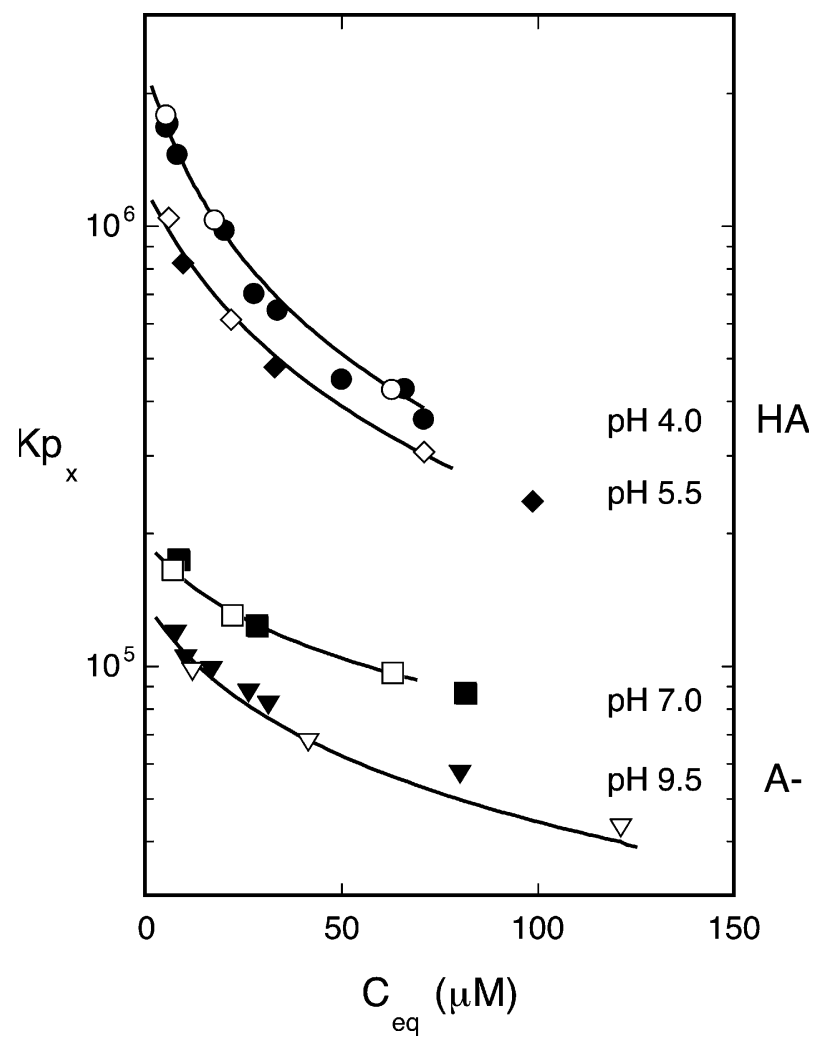

Fig. 5. Experimental results illustrating the independence of the partition coefficient of un-ionized (HA) and ionized (A-) forms of $\mathrm{TeCP}$ on the presence of carbonyl groups of membrane lipids. Plots of measured partition coefficient as a function of equilibrium concentration of TeCP for lipid DODPC (empty symbols, carbonyl groups absent) and DOPC (filled symbols, carbonyl groups present). Measurements were done at pH $4.0 \& 4.1$ (circles), 5.5 (diamonds), 7.0 (squares) and 9.5 (triangles). The curves are theoretical, $K p_{\mathrm{x}}(\mathrm{th})$ for DODPC, based on least-squares fits of all $K p_{\mathrm{x}}\left(\right.$ exper) data at each $\mathrm{pH}$. The theoretical curves of $K p_{\mathrm{x}}(\mathrm{th})$ vs equilibrium concentration of TeCP included Eqs. (24a), (24b), (25a), (25b), (26), (30) and the left side of Eq. (33). The curves were calculated using $K p_{\mathrm{x} 0}^{\mathrm{HA}}=2.44 \times 10^{6}$, for $\mathrm{pH} 5.5$ using $K p_{\mathrm{x} 0}^{\mathrm{HA}}=2.35 \times 10^{6}$, for $\mathrm{pH} 7.0$ using $K p_{\mathrm{x} 0}^{\mathrm{A}}=0.141 \times 10^{6}$, and for pH 9.5 using $K p_{\mathrm{x} 0}^{\mathrm{A}}=0.138 \times 10^{6}$. Partition coefficients obtained for DODPC are also applicable to DOPC results.

bilayer result in the more positive dipolar potential in phosphatidylcholine membranes (Simon, et al., 1992; Cafiso 1995). The Gibbs free energy profile of the water-lipid interface is complex and the dipolar potential contributes to it. There are several constituents of the dipolar potential, (1) oriented water molecules at the water-membrane interface, (2)-P-N ${ }^{+}$dipole of the phospholipid head group, (3) dipoles of phosphorus-to-oxygen groups of the phosphate group (Diaz, et al., 2001), and (4) carbonyl groups in the ester link of acyl chains (Gawrisch, et al. 1992).

Partition coefficients of (HA) and (A-) species provide information on Gibbs free energy of these species in the membrane. The ratio of partition coefficients 
Table 4. Effect of dipolar layer on partition. Least-squares-fit values of the infinite dilution partition coefficient, $K \mathrm{p}_{\mathrm{x} 0}$, of the un-ionized (HA) and ionized (A-) species of TeCP

\begin{tabular}{|c|c|c|c|c|c|c|}
\hline Lipid & $\mathrm{pH}$ & $N$ & $\chi^{2}$ & $K \mathrm{p}_{\mathrm{x} 0}$ & $\mathrm{TeCP}$ & $r^{2}$ \\
\hline \multicolumn{7}{|l|}{$(A)$} \\
\hline \multirow[t]{4}{*}{ DODPC (carbonyl group absent) } & 4.0 & 3 & 0.10 & $(2.44 \pm 0.16) \times 10^{6}$ & HA & \\
\hline & 5.5 & 3 & 2.2 & $(2.35 \pm 0.09) \times 10^{6}$ & HA & \\
\hline & 7.0 & 3 & 0.15 & $(0.141 \pm 0.009) \times 10^{6}$ & A- & \\
\hline & 9.5 & 3 & 3.9 & $(0.138 \pm 0.006) \times 10^{6}$ & A- & \\
\hline \multirow[t]{4}{*}{ DOPC (carbonyl group present) } & 4.1 & 9 & 7.0 & $(2.35 \pm 0.05) \times 10^{6}$ & HA & \\
\hline & 5.5 & 3 & 0.92 & $(2.16 \pm 0.09) \times 10^{6}$ & HA & \\
\hline & 7.0 & 3 & 0.48 & $(0.168 \pm 0.013) \times 10^{6}$ & A- & \\
\hline & 9.5 & 6 & 4.9 & $(0.149 \pm 0.005) \times 10^{6}$ & A- & \\
\hline \multicolumn{7}{|l|}{ (B) } \\
\hline DODPC (carbonyl group absent) & 4.0 & 3 & 0.12 & $(2.36 \pm 0.18) \times 10^{6}$ & HA & 0.999 \\
\hline DODPC (carbonyl group absent) & 9.5 & 3 & 0.35 & $(0.120 \pm 0.010) \times 10^{6}$ & A- & 0.996 \\
\hline DOPC (carbonyl group present) & 4.1 & 9 & 63 & $(1.86 \pm 0.08) \times 10^{6}$ & HA & 0.94 \\
\hline DOPC (carbonyl group present) & 9.5 & 6 & 0.58 & $(0.131 \pm 0.009) \times 10^{6}$ & A- & 0.99 \\
\hline
\end{tabular}

(A) Results obtained from the dependence of $K \mathrm{p}_{\mathrm{x}}$ on the aqueous equilibrium concentration of TeCP. The least-squares-fit method includes Eqs. (5), (24a), (24b), (25a), (25b), (26), (30) and (33).

(B) Results obtained from the dependence of $K \mathrm{p}_{\mathrm{x}}$ on the mole fraction of bound TeCP

$$
\frac{K p_{\mathrm{x} 0}(D O D P C)}{K p_{\mathrm{x} 0}(D O P C)}=\exp \left(-\frac{\Delta G_{\text {carbonyl }}}{R T}\right)
$$

is a sensitive measure of the change of free energy at the membrane partition site associated with the absence of carbonyl groups in DODPC. A factor of two in the ratio of partition coefficients, which would be easily detectable in our study, corresponds to a change of free energy of $1.7 \mathrm{~kJ} / \mathrm{mol}$ or $18 \mathrm{meV}$. For ionized $\mathrm{TeCP}$, the change of partition coefficients by a factor of two would correspond to a dipolar potential of $18 \mathrm{mV}$. This is substantially less than the carbonyl dipole potential difference that was estimated to be 100-120 mV (Gawrisch, et al. 1992).

The fact that partition coefficient of ionized $\mathrm{TeCP}$ is the same for DODPC and DOPC membranes offers two possible explanations: (1) the presence of (A-) species causes dipolar disorder within the interface so that dipole moments of carbonyl groups in the vicinity of (A-) are not oriented inward as in an unperturbed bilayer. It was shown that multiple charged ions (Ermakov, et al. 2001) and thyroid hormones incorporated into lipid bilayer (Isse, et al. 2003) perturb dipolar organization of the interface. Another possibility is (2) that the center of charge of ionized TeCP, which is close to oxygen, is either in front or at the edge of the carbonyl dipolar layer, so that it does not sense the carbonyl dipolar potential contribution of DOPC membrane. This explanation is somewhat tenuous since it is not known what is the contribution from water of hydration (Diaz, et al. 1999). Both alternatives assume that ionized $\mathrm{TeCP}$ is localized within the waterlipid interface of membrane. This assumption is supported by earlier solvatochromic studies of membrane-bound ionized pentachlorophenol, regarded here as a reference compound similar to TeCP (Fig. 6). The UV spectral shifts of membranebound ionized pentachlorophenol were due to hydrogen bonding of (A-) to water penetrating into the membrane interface (Smejtek, et al. 1987). It should be mentioned that no such shifts were detected for un-ionized pentachlorophenol. Due to the similar structure and properties of $\mathrm{TeCP}$ and pentachlorophenol, and the absence of a carbonyl-group effect, we conclude that ionized TeCP is localized within the interface layer in the region of partial water penetration into the membrane. Thus the observed insensitivity of the partition coefficient to the presence or absence of a carbonyl group of lipids is associated with localization of (A-). In contrast to ionized TeCP, the lipophilic anion tetraphenylborate and lipophilic cation tetraphenylphosphonium were found to respond to dipolar potential (Flewelling \& Hubbell 1986). The apparent contradiction of our finding i.e., null effect of carbonyl dipoles, with those of Flewelling and Hubbell, can be resolved by considering depth of localization and hydrogen bonding properties of TeCP anion and lipophilic ions. Lipophilic ions must be located deeper in the membrane, below the layer of carbonyl dipoles, in part due to the absence of hydrogen bonding to water. It follows from the similarity of hydrogen bonding properties of $\mathrm{TeCP}$ and pentachlorophenol that relative to tetraphenylborate the center of charge of ionized $\mathrm{TeCP}$ is expected to be closer to aqueous phase and thus it is insensitive to dipolar potential.

The partition coefficient of uncharged $\mathrm{TeCP}$ is typically 20 times greater than that of ionized species. (HA) species are more hydrophobic and therefore expected to partition deeper into the bilayer. It is 
<smiles>Oc1c(Cl)cc(Cl)c(Cl)c1Cl</smiles>

2,3,4,6-TeCP

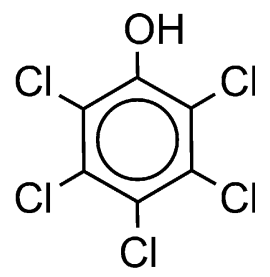

PCP
Fig. 6. Molecular structure of un-ionized 2,3,4,6-tetrachlorophenol, TeCP, and 2,3,4,5,6-pentachlorophenol, PCP, regarded as a reference compound.

assumed that un-ionized TeCP is present in the membrane below the water penetration layer since solvatochromic shifts of un-ionized pentachlorophenol were not observed (Smejtek, Barstad \& Wang, 1987). It is very likely that the hydroxyl group of $\mathrm{TeCP}$ forms hydrogen bonds with oxygens of phosphate groups of DOPC and DODPC rather than with water. The chloro-substituted phenyl ring of TeCP is closer to the center of the bilayer than the HO-group and it is aligned with the upper segment of oriented acyl chains of phospholipids. From the comparison of octanol/water and liposome/water partition coefficients of substituted phenols, and use of steric arguments, Miyoshi and coworkers (Miyoshi, et al. 1987) also concluded that phenols reside within the membrane/water interface. The above localization of (HA) and (A-) species would result in independence of the partition coefficient of TeCP on the length of acyl chains of lipids, which was experimentally demonstrated.

Pertinent to the problem of localization of the partition site of TeCP are the results of a recent molecular-dynamics study of localization of pentachlorophenol in PC membranes (Mukhopadhyay, et al. 2004). The relevance of this study is that TeCP and pentachlorophenol are structurally similar (Fig. 6). Results of the computer simulation study of distribution of pentachlorophenol between water and palmitoyl-oleoyl-phosphatidylethanolamine and palmitoyl-oleoyl-phosphatidylcholine membranes have indicated that un-ionized pentachlorophenol is preferentially localized in the region between the double bond on one of the acyl chains and the layer of carbonyl groups. This is also the expected region of localization of un-ionized TeCP. Furthermore, the simulations confirmed that the phenyl ring of pentachlorophenol is oriented parallel to acyl chains and the pentachlorophenol hydroxyl group interacts via hydrogen bonds with lipid oxygen and water. Results of the molecular-dynamics study (Mukhopadhyay, et al. 2004) are relevant to partitioning of TeCP, and are consistent with the partition properties of $\mathrm{TeCP}$ reported in this work.

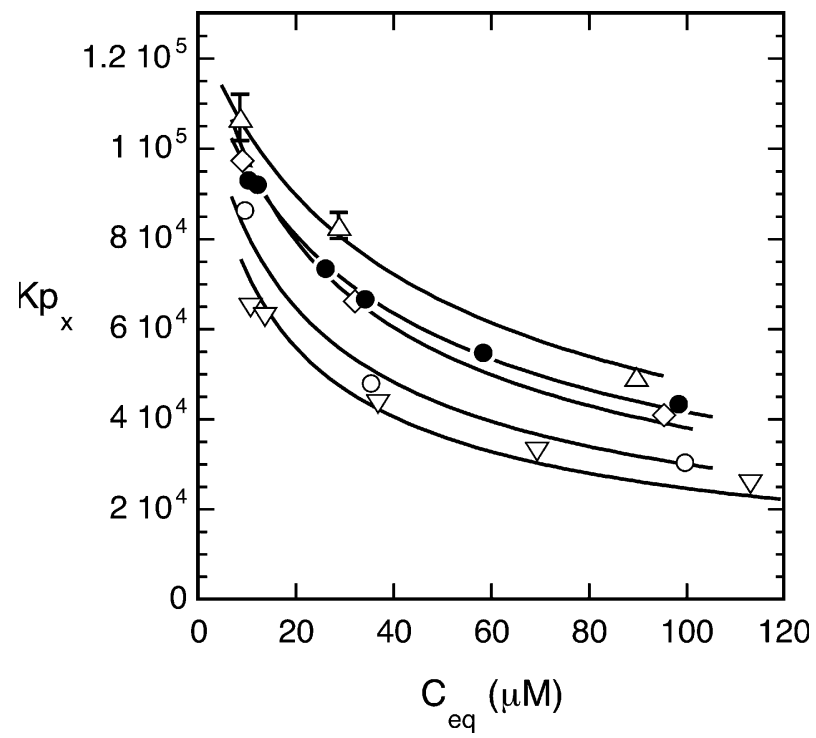

Fig. 7. Experimental results illustrating the effect of ionic strength on partitioning of ionized TeCP between water and egg-PC liposome membrane. Ionic strength was controlled by concentration of KCI: $10 \mathrm{mM}(\nabla), 20 \mathrm{mM}(\bigcirc), 50 \mathrm{mM}(\diamond), 100 \mathrm{mM}(\bullet)$, and 200 $\mathrm{mM}(\triangle)$. The $\mathrm{pH} 9.4$ buffer was $10.0 \mathrm{mM}$ CHES. As the ionic strength increases, the repulsive electrostatic interactions between ionized TeCP in membrane and free TeCP ions in water decrease, resulting in greater $K p_{\mathrm{x}}$. The solid curves are theoretical obtained using Eqs. (24b), (25b), (26), (30) and the left side of Eq. (31) for least-square-fit values of $K p_{\mathrm{x} 0}$ listed in Table 5.

Effect of Ionic Strength on Partitioning of TeCP Between Water and Egg-PC Membranes

Free ions in liposome suspension reduce electrostatic interactions between membrane and partitioning solutes. As expected, we have found no effect on partition of un-ionized TeCP between water and PC liposomes. In the case of ionized TeCP, the uncharged PC biiayer becomes negatively charged due to the presence of (A-) in the membrane. The electrostatic repulsion between the negatively charged biiayer surface and ionized TeCP in water reduces the local concentration of $\mathrm{TeCP}$ at the aqueous portion of the membrane/water interface that results in reduced partition into the membrane. The effect is manifested by reduced $K p_{\mathrm{x}}$. At higher ionic strength the charges on the bilayer are screened by counterions to a greater degree, resulting in less negative surface electrostatic potential $\psi$, which results in higher local concentration of ionized TeCP at the interface. Thus, at higher ionic strength, partitioning of ionized TeCP is greater, resulting in greater $K p_{\mathrm{x}}$. In our model the effect of electrostatic interactions is described by Eqs. (26), (29) and (24b).

Experimental results of the effect of ionic strength are shown in Fig. 7, illustrates the dependence of $K p_{\mathrm{x}}$ on the equilibrium concentration of ionized TeCP. In this study, the ionic strength was 
Table 5. Least-squares-fit values of the infinite dilution partition coefficient, $K \mathrm{p}_{\mathrm{x} 0}$ of the ionized (A-) species of TeCP.

\begin{tabular}{lllll}
\hline $\mathrm{KCl}, \mathrm{mM}$ & Ionic strength, $\mathrm{M}$ & $n$ & $\chi^{2}$ & Kpxo $^{\mathrm{A}}$ \\
\hline 200 & 0.205 & 3 & 0.35 & $(0.126 \pm 0.008) \times 10^{6}$ \\
100 & 0.105 & 6 & 1.4 & $(0.121 \pm 0.004) \times 10^{6}$ \\
50.0 & 55.0 & 3 & 1.1 & $(0.135 \pm 0.007) \times 10^{6}$ \\
20.0 & 25.0 & 3 & 2.3 & $(0.119 \pm 0.006) \times 10^{6}$ \\
10.0 & 11.5 & 5 & 13 & $(0.118 \pm 0.005) \times 10^{6}$
\end{tabular}

Results obtained from the dependence of $K \mathrm{p}_{\mathrm{x}}$ on the ionic strength of aqueous phase. The least-squares-fit method includes Eqs. (5), (24b), (25b), (26), (30) and (31) assuming partition site area $A_{\mathrm{s}}=0.65 \mathrm{~nm}^{2}$.

controlled by variable $\mathrm{KCl}$ concentration $(10,20,50$, 100 and $200 \mathrm{mM}$ ) in $10 \mathrm{~mm}$ CHES buffer. The successive $K p_{\mathrm{x}}$ isotherms demonstrate that with increasing ionic strength the magnitude of the partition coefficient increases due to electrostatic screening of the bilayer surface charged by the presence of ionized $\mathrm{TeCP}$ in the membrane. The solid curves depict the least-squares-fit of the model to each experimental data point, yielding the infinite dilution partition coefficient $K p_{\mathrm{x} 0}^{\mathrm{A}}$. Results are summarized in Table 5. We obtained very similar values of the infinite dilution partition coefficients, demonstrating that Eqs. (26), (29) and (24b) correctly account for electrostatic interactions in this study and demonstrating the possibility to obtain a thermodynamically meaningful partition coefficient of ionized TeCP, $K p_{\mathrm{x} 0}^{\mathrm{A}}$.

Partitioning of TeCP between Water and Sarcoplasmic Reticulum Membrane

We have used sarcoplasmic reticulum membrane as a model biomembrane to explore the effect of membrane proteins and biomembrane structure on partition. Two studies were done, one with SR vesicles and one with egg-PC single-layered liposomes. Since we determined the amount of lipids in both the SR and lipid vesicles, the partition coefficients for SR are "normalized" to lipids present in SR. In this way the results for SR can be directly compared with data from liposomes. In order to cover the complete range of $\mathrm{TeCP}$ ionization, from (HA) to (A-), the measurements with SR were performed at $\mathrm{pH}$ 4.1, 5.6, 6.7 and 9.3. The measured partition coefficient for the lowest and the highest $\mathrm{pH}$ are most representative for the un-ionized and ionized TeCP because the contribution of minority TeCP species is minimized.

Experimental partition coefficients for SR vesicles and egg-PC liposomes are shown in Fig. 8. The solid curves illustrate the prediction of the model for SR and the broken curves, for egg-PC liposomes. The infinite dilution partition coefficients were obtained from fit of the partition model to the dependence of $K p_{\mathrm{x}}$ on aqueous equilibrium concentration and to the dependence of $K p_{\mathrm{x}}$ on mole fraction of membrane bound $\mathrm{TeCP}$. Both are very similar. The experimental results are summarized in Table $6 A, B$.

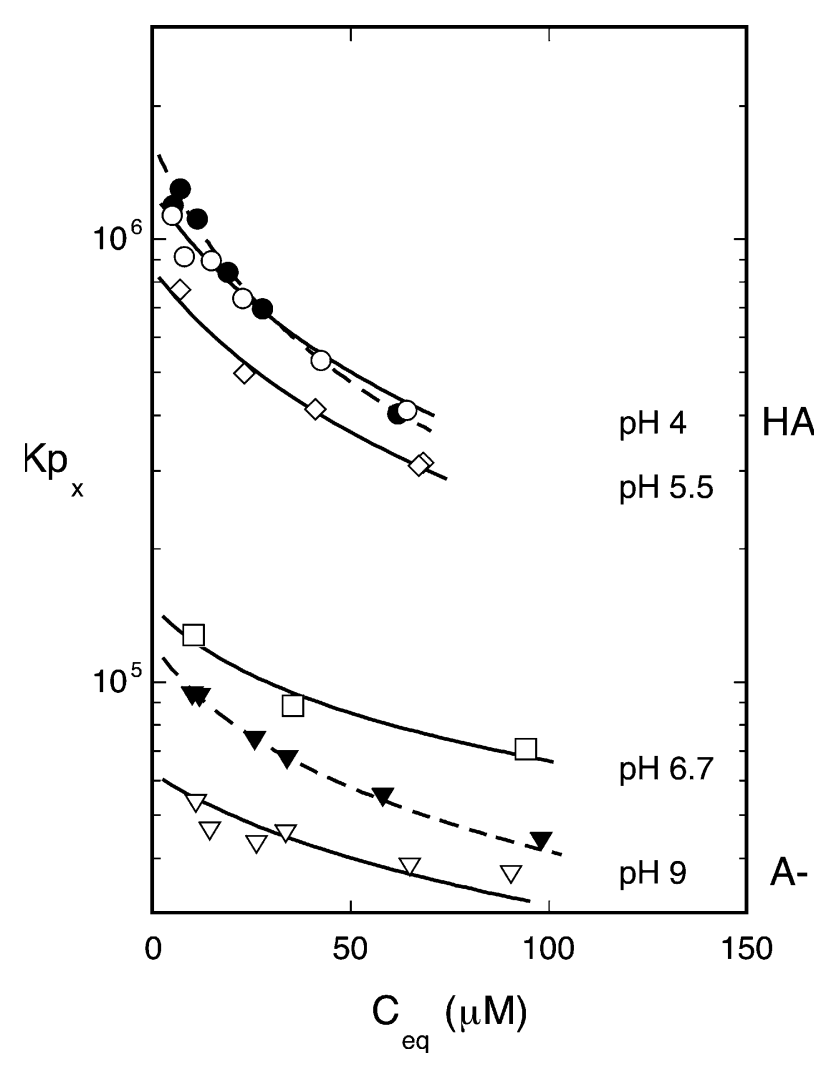

Fig. 8. Comparison of partition characteristics of TeCP for sarcoplasmic reticulum (SR) and egg-PC membranes. Plots of the partition coefficient as a function of equilibrium concentration of $\mathrm{TeCP}$ and $\mathrm{pH}$ for SR (empty symbols) and egg-PC bilayer (filled symbols). Partition measurements with SR were done at $\mathrm{pH} 4.0$ \& $4.1(\bigcirc), 5.5(\diamond), 6.7(\square)$ and $9.0 \& 9.4(\nabla, \nabla)$. The solid curves illustrate the least-squares fit of the partition model for SR, the broken curves for egg-PC. $K p_{\mathrm{x} 0}^{\mathrm{HA}}(\mathrm{SR}) \approx K p_{\mathrm{x} 0}^{\mathrm{HA}}($ egg-PC), whereas $K p_{\mathrm{x} 0}^{\mathrm{A}}(\mathrm{SR})<K p_{\mathrm{x} 0}^{\mathrm{A}}($ egg-PC), values given in Table $6 A, B$.

Results of this comparative study indicate that partition coefficient of un-ionized TeCP for SR and egg-PC membranes are approximately the same. In the simplest case, it indicates that the biomembrane structure and presence of protein in SR membrane have no effect on partitioning and that un-ionized $\mathrm{TeCP}$ accumulates in the lipid matrix of the biomembrane, consistent with Herbette's formulation of a membrane-mediated mechanism of action of amphipilic molecules (Herbette \& Gruner 1987). 
Table 6. Comparison of partition properties of TeCP for sarcoplasmic reticulum and egg-PC membranes. Least-squares-fit values of the infinite dilution partition coefficient, $K \mathrm{p}_{\mathrm{x} 0}$ of the un-ionized (HA) and ionized (A-) species of TeCP.

\begin{tabular}{|c|c|c|c|c|c|c|}
\hline Membrane & $\mathrm{pH}$ & $n$ & $\chi^{2}$ & $K \mathrm{p}_{\mathrm{x} 0}$ & $\mathrm{TeCP}$ & $r^{2}$ \\
\hline \multicolumn{7}{|l|}{$(A)$} \\
\hline \multirow[t]{3}{*}{ Sarcoplasmic reticulum } & 4.1 & 6 & 7.5 & $(1.60 \pm 0.14) \times 10^{6}$ & HA & \\
\hline & 5.5 & 3 & 0.88 & $(2.07 \pm 0.26) \times 10^{6}$ & HA & \\
\hline & 9.5 & 3 & 13 & $(0.0867 \pm 0.0032) \times 10^{6}$ & A- & \\
\hline \multirow{2}{*}{ Egg-PC (reference) } & 4.0 & 6 & 1.4 & $(1.81 \pm 0.06) \times 10^{6}$ & HA & \\
\hline & 9.4 & 6 & 1.4 & $(0.121 \pm 0.004) \times 10^{6}$ & A- & \\
\hline \multicolumn{7}{|c|}{ 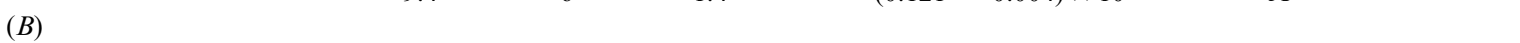 } \\
\hline Sarcoplasmic reticulum & 4.1 & 6 & 0.61 & $(1.27 \pm 0.14) \times 10^{6}$ & HA & 0.949 \\
\hline Sarcoplasmic reticulum & 9.0 & 6 & 2.3 & $(0.0534 \pm 0.0033) \times 10^{6}$ & A- & 0.819 \\
\hline Egg-PC (reference) & 4.0 & 6 & 1.7 & $(1.68 \pm 0.10) \times 10^{6}$ & HA & 0.93 \\
\hline Egg-PC (reference) & 9.4 & 6 & 0.32 & $(0.115 \pm 0.007) \times 10^{6}$ & A- & 0.99 \\
\hline
\end{tabular}

(A) Results obtained from the dependence of $K \mathrm{p}_{\mathrm{x}}$ on the aqueous equilibrium concentration of TeCP. The least-squares-fit method includes Eqs. (5), (24a), (24b), (25a), (25b), (26), (30) and (33).

(B) Results obtained from the dependence of $K \mathrm{p}_{\mathrm{x}}$ on the mole fraction of bound TeCP. (5), (24a), (24b), (26), (30) and (33).

There is, however, a singular possibility that un-ionized TeCP partitions into SR proteins and that at the same time, this gain is compensated by reduced partitioning into lipid matrix. Favoring the more probable alternative, we conclude that the partition coefficient of un-ionized TeCP for SR membrane is about the same as that for egg-PC. As follows from data in Table $6 A, B$, the partition coefficient of ionized TeCP for SR membrane is smaller than that for egg-PC. We believe that the difference is due to electrostatic repulsion of ionized TeCP from the surface of native SR since the electrophoretic mobility of SR vesicles is negative (Arrio, et al. 1984). This effect cannot be accounted for at this time because the electrostatic potential of SR membrane is not known. According to our results (not published), the electrophoretic mobility of SR at 10 $\mathrm{mM}$ ionic strength was $\mathrm{pH}$-independent in the $\mathrm{pH}$ range $7-10$, having an average value of $-1.44 \pm 0.06$ mobility units $\left(\mu \mathrm{m} \mathrm{s} \mathrm{s}^{-1} \mathrm{~V}^{-1} \mathrm{~cm}\right)$. The magnitude of the electrostatic potential at the surface of SR is not known due to the presence of a hydrodynamic retardation effect at the SR surface. If the mobility of SR vesicles is analyzed using the conventional mobility model, in which SR is treated as a smooth hard particle (Arrio, et al. 1984), the electrostatic potential of SR surface used in the fit of SR partition data yields a partition coefficient of ionized TeCP smaller than that for egg-PC membrane. It should be mentioned that a hard-particle model of SR may underestimate the electrostatic potential at the surface of the lipid matrix of SR membrane where sorption of ionized TeCP takes place. It is likely that the electrostatic potential at the surface of the lipid matrix of SR membrane is more negative than that estimated from conventional electrophoretic mobility theory and that $K p_{\mathrm{x} 0}$ of ionized TeCP of SR matrix and egg-PC bilayers are approximately the same. This point can be resolved in future studies. Our major conclusion is that in the case of SR, the structure of biomembrane and the presence of membrane proteins do not alter partitioning of TeCP into the biomembrane and that $\mathrm{TeCP}$ resides entirely within the lipid matrix of SR. Our data suggest that TeCP does not partition into SR proteins. This observation is important since chlorinated phenols, including TeCP, are expected to bind to bovine serum albumin (Gulden, et al. 2003).

\section{Summary and Conclusions}

We have studied partitioning of a small amphiphilic molecule, 2,3,4,6-tetrachlorophenol, because its hydrophobicity makes it possible to study partitioning of both un-ionized and ionized species of almost identical structure. We have used the mole fraction partition coefficient as the most suitable measure of partitioning because the volume of membrane in which these molecules are preferentially localized is not known. We have studied (a) the effect of the length of acyl chains of lipids using water-alkane partition coefficients of un-ionized TeCP as the reference; (b) the role of dipolar potential of lipid bilayer in partition; (c) the effect of ionic strength on partitioning of ionized TeCP, and (d) the effect of structure and proteins of biological membranes by comparing partition properties of TeCP for sarcoplasmic reticulum and egg-PC membrane.

We have described an experimental protocol for the accurate measurement of partition coefficients using equilibrium dialysis. The studies of partitioning between water and lipid membranes were done using single-layered lipid vesicles made from lipids with acyl chains of 16 to 24 carbons in length. The measured partition coefficients were dependent on the aqueous concentration of $\mathrm{TeCP}$ and, in the case of ionized TeCP, on ionic strength of aqueous phase. Experimental data were analyzed in terms of a par- 
tition model that accounts for the saturation of partition sites in the membrane, and electrostatic interactions between charged membrane and ionized $\mathrm{TeCP}$ in the aqueous portion of the membrane/water interface. The model includes co-partitioning of unionized and ionized TeCP and describes partition results over a wide range of $\mathrm{pH}$.

From the fit of the partition model to measured partition coefficients we obtained infinite dilution partition coefficients, $K p_{\mathrm{x} 0}$, that have thermodynamic significance. We have found that, contrary to expectation, $K p_{\mathrm{x} 0}$ of un-ionized and ionized TeCP are independent of the length of acyl chains of lipids, whereas for alkanes $K p_{\mathrm{x} 0}$ of un-ionized. TeCP increases with chain length. For un-ionized and ionized TeCP the representative values of $K p_{\mathrm{x} 0}$ are $2.6 \times 10^{6}$ and $0.15 \times 10^{6}$, respectively.

We have explored the effect of carbonyl groups of lipids on partitioning of un-ionized and ionized TeCP using DODPC (dioctadecenylphosphatidylcholine) and DOPC (dioleoylphosphatidylcholine) membranes. These lipids differ only by the presence of a carbonyl group in the linkage of acyl chains to polar head group. We found that $K p_{\mathrm{x} 0}$ for both forms of TeCP and both DODPC and DOPC membranes are the same. In contrast to membrane transport and partitioning of lipophilic ions, the magnitude of the dipolar potential associated with carbonyl groups has no effect on partitioning. The invariance of $K p_{\mathrm{x} 0}$ to the length of acyl chains and to the presence of carbonyl groups of lipids can be understood if the un-ionized TeCP is localized in the upper segment of acyl chains and if the center of charge on ionized TeCP does not sense the dipolar potential of the carbonyl group. This may be due to dipolar disorder induced by the ionized $\mathrm{TeCP}$ or due to position of the center of charge on the aqueous side of the carbonyl dipolar layer.

We have explored the question of similarity of partitioning of TeCP in lipid bilayer and sarcoplasmic reticulum membrane. It turned out that partition coefficients for transfer of TeCP from water into sarcoplasmic reticulum membrane are very similar to those for egg-PC bilayers. It means that in case of sarcoplasmic reticulum, the structure of biomembrane and the presence of proteins have little if any effect on partitioning of amphiphilic TeCP and that TeCP partitions mainly into the lipid matrix of sarcoplasmic reticulum membrane.

We thank Professor Jon Abramson for discussions and for making this study possible by providing us with sarcoplasmic reticulum vesicles. We are also grateful to Professor Petr Paucek for advice and guidance with the preparation of single-layered liposomes.

\section{References}

Arrio, B., Johannin, G., Carrette, A., Chevallier, G., Brethes, D. 1984. Electrokinetic and hydrodynamic properties of sarco- plasmic reticulum vesicles: A study by laser Doppler electrophoresis and quasielastic light scattering. Arch. Biochem. Biophys 228:220-229

Ben-Naim, A. 1979. Reply to C. Tanford's comments concerning standard states in the thermodynamics of transfer. J. Phys. Chem. 83: 1803

Bevington, P., Robinson, D. 2003. Data Reduction and Error Analysis for the Physical Sciences. McGraw-Hill, New York.

Cafiso, D.S. 1995. Influence of charges and dipoles on macromolecular adsorption and permeability. In: Permeability and Stability of Lipid Bilayers. Disalvo, E.A., Simon, S.A. eds. pp 179196, CRP Press, Boca Raton, Florida

Cantor, R.S 2001. Breaking the Meyer-Overton rule: predicted effects of varying stiffness and interfacial activity on the intrinsic property of anesthetics. Biophys. J. 80:2284-2297

Chen, P., Toribara, T., Warner, H. 1956. Microdetermination of phosphorus. Anal. Chem. 28:1756-1758

Diaz, S., Amalfa, F., de Biodi Lopez, A.C., Disalvo, E.A 1999. Effect of water polarized at the carbonyl groups of phosphatidylcholines on the dipole potential of lipid bilayers. Langmuir 15:5179-5182

Diaz, S., Lairion, F., Arroyo, J., de Bio Lopez, A.C., Disalvo, E.A 2001. Contribution of phosphate groups to the dipole potential of dimyristoylphosphatidylcholine membranes. Langmuir 17:852-855

Ermakov, Y.A., Averbakh, A.Z., Yusipovich, A.I., Sukharev, S. 2001. Dipole potentials indicate restructuring of the membrane interface induced by gadolinium and beryllium ions. Biophys. J. 80:1851-1862

Escher, B.I., Hunziker, R., Schwarzenbach, R.P 1999. Kinetic model to describe the intrinsic uncoupling activity of substituted phenols in energy transducing membranes. Environ. Sci. Technol. 33:560-570

Escher, B.I., Schwarzenbach, R.P., Westall, J.C 2000. Evaluation of liposome-water partitioning of organic acids and bases. 1. Development of a sorption model. Environ. Sci. Technol. 34:3954-3961

Escher, B.I., Snozzi, M., Schwarzenbach, R.P 1996. Uptake, speciation, and uncoupling activity of substituted phenols in energy transducing membranes. Environ. Sci. Technol. 30:30713079

Flewelling, R.F., Hubbell, W.L 1986. The membrane dipole potential in a total membrane potential model. Applications to hydrophobic ion interactions with membranes. Biophys. J. 49:541-552

Gawrisch, K., Ruston, D., Zimmerberg, J., Parsegian, V.A., Rand, R.P., Fuller, N. 1992. Membrane dipole potentials, hydration forces, and the ordering of water at membrane surfaces. Biophys. J. 61:1213-1223

Gulden, M., Morchel, S., Tahan, S., Seibert, H. 2003. Impact of protein binding on the availability and cytotoxic potency of organochlorine pesticides and chlorophenols in vitro. Toxicology 175:201-213

Herbette, L.G., Chester, D.W., Rhodes, D.G 1986. Structural analysis of drug molecules in biological membranes. Biophys. J. 49:91-94

Herbette, L.G., Gruner, S.M 1987. The role of the lipid bilayer in amphiphile-membrane/receptor interactions: a unifying hypothesis. Dev. Cardiovasc. Med. 68:353-365

Holloway, P.W 1973. A simple procedure for removal of Triton X100 from protein samples. Anal. Biochem. 53:304-308

Isse, B., Fidelio, G., Farias, R.N 2003. Thyroid hormones affect the membrane dipolar organization. Is it a general event in their non-genomic action? J. Membrane Biol. 191:209-213

Kleinzeller, A. 1999. Charles Ernest Overton's concept of a cell membrane. Curr. Top. Membr. 48:1-22 
Kramer, S. 2001. Liposome/water partitioning: Theory, techniques, and applications. In: Pharmacokinetic Optimization in Drug Research: Biological, Physicochemical, and Computational Strategies. Testa, B., Waterbeemd, V., Folkers, G., Guy, R. eds. pp 401-428, Verlag Helvetica Chimica Acta, Zurich

Miyoshi, H., Maeda, H., Tokutake, N., Fujita, T. 1987. Quantitative analysis of partition behavior of substituted phenols from aqueous phase into liposomes made of lecithin and various lipids. Bull. Chem. Soc. Jpn. 60:4357-4362

Mukhopadhyay, P., Vogel, H., Tieleman, D. 2004. Distribution of pentachlorophenol in phospholipid bilayers: A molecular dynamics study. Biophys. J. 86:337-345

Paucek, P., Mironova, G., Mahdi, F., Beavis, A.D., Woldegiorgis, G., Garlid, K. 1992. Reconstitution and partial purification of the glibenclamide-sensitive, ATP-dependent $\mathrm{K}^{+}$channel from rat liver and beef heart mitochondria. J. Biol. Chem. 267:2606226069

Plemper van Balen, G., Martinet, C., Caron, G., Bouchard, G., Reist, M., Carrupt, P., Fruttero, R., Gasco, A., Testa, B. 2004. Liposome/water lipophilicity: Methods, information content, and pharmaceutical applications. Medicinal Res. Rev. 24:299-324

Rigaud, J., Levy, D., Mosser, G., Lambert, O. 1998. Detergent removal by non-polar polystyrene beads. Application to membrane protein reconstitution and two-dimensional crystallization. Eur. Biophys. J. 27:305-319

Rokitskaya, T.I., Kotova, E.A., Antonenko, Y.N 2002. Membrane dipole potential modulates proton conductance through gramicidin channel: Movement of negative ionic defects inside the channel. Biophys. J. 82:865-873

Santos, N.C., Prieto, M., Castanho, M.A.R.B 2003. Quantifying molecular partition into model systems of biomembranes: an emphasis on optical spectroscopic methods. Biochim. Biophys. Acta 1612:123-135
Schellenberg, K., Leuenberger, C., Schwarzenbach, R. 1984. Sorption of chlorinated phenols by natural sediments and aquifer materials. Environ. Sci. Technol. 18:652-657

Seydel, J.K 2002. Octanol-water partitioning versus partitioning into membranes. Drug-membrane interactions; analysis, drug distribution,. modeling 15:35-50

Simon, S.A., Mclntosh, T.J., Magid, A.D., Needham, D. 1992. Modulation of the interbilayer hydration pressure by the addition of dipoles at the hydrocarbon/water interface. Biophys. J. 61:786-799

Smejtek, P. 1995. Permeability of lipophilic ions across lipid bilayers. In: Permeability and Stability of Lipid Bilayers. Disalvo, E.A., Simon, S.A. eds. pp 197-240, CRC Press, Boca Raton

Smejtek, P., Wang, S. 1990. Adsorption to dipalmitoylphosphatidylcholine membranes in gel and fluid state: pentachlorophenolate, dipicrylamine and tetraphenylborate. Biophys. J. 58:1285-1294

Smejtek, P., Word, R.C 2004. Environmental swap energy and role of configurational entropy in transfer of small molecules from water into alkanes. J. Chem. Phys. 120:1383-1394

Smejtek, P., Barstad, A., Wang, S. 1987. Dielectric properties of adsorption/ionization site of pentachlorophenol (PCP) in lipid membranes. Biochim. Biophys. Acta 902:109-127

Starzak, M. 1984. The physical chemistry of membranes. Academic Press, New York,

Tanford, C. 1979. Standard states in the thermodynamics of transfer. J. Phys. Chem. 83:1802-1803

White, S.H., Wimley, W.C 1999. Membrane protein folding and stability: Physical principles. Annu. Rev. Biophys. Biomol. Struct. 28:319-365

Xia, R., Webb, J.A., Gnall, L.L.M., Cutler, K., Abramson, J.J 2003. Skeletal muscle sarcoplasmic reticulum contains a NADH-dependent oxidase that generates superoxides. Am. J. Physiol. 285:C215-C221 